\title{
EXISTENCE OF DIRICHLET FINITE HARMONIC MEASURES ON EUCLIDEAN BALLS
}

\author{
MITSURU NAKAI \\ To Professor Makoto Ohtsuka on his seventieth birthday
}

Divide the ideal boundary of a noncompact Riemannian manifold $M$ into two parts $\delta_{0}$ and $\delta_{1}$. Viewing that $M$ is surrounded by two conducting electrodes $\delta_{0}$ and $\delta_{1}$, we ask whether $\left(M ; \delta_{0}, \delta_{1}\right)$ functions as a condenser in the sense that the unit electrostatic potential difference between two electrodes is produced by putting a charge of finite energy on one electrode when the other is grounded. The generalized condenser problem asks whether there exists a subdivision $\delta_{0} \cup \delta_{1}$ of the ideal boundary of $M$ such that $\left(M ; \delta_{0}, \delta_{1}\right)$ functions as a condenser.

Mathematically the problem has two equivalent formulations one of which is in the geometric and the other in the analytic form. In the geometric formulation the problem is negatively settled if and only if the linear Royden harmonic boundary $\Delta(M)$ of $M$ is connected. In the analytic formulation the problem is also negatively settled if and only if every Dirichlet finite harmonic measure on $M$ reduces to a constant on $M$. A fairy general discussion of the generalized condenser problem for Riemannian manifolds $M$ was carried out in [22] one of whose consequences is that the Royden harmonic boundary $\Delta\left(B^{n}\right)$ of the Euclidean unit ball $B^{n}$ in the Euclidean space $R^{n}$ of dimension $n \geq 2$ is connected which generalizes the classical result in the complex function theory that $\Delta\left(B^{2}\right)$ is connected (cf. e.g. [3], [1], [12], [10], [21], [25], etc.).

In this paper we discuss the above generalized condenser problem for the Euclidean ball $B^{n}$ of dimension $n \geqq 2$ in a broader potential theoretic setting that the underlying harmonic structure is given by the $p$-Laplace equation

$$
-\nabla \cdot\left(|\nabla u|^{p-2} \nabla u\right)=0 \quad(1<p \leqq n),
$$

the solutions of which are the so-called $p$-harmonic functions. Here the exponent $p$

Received February 1, 1993.

This work was partly supported by Grant-in-Aid for Scientific Research, No. 04640050 , Japanese Ministry of Education, Science and Culture. 
is restricted to $1<p \leqq n$ since otherwise the structure reduces to a triviality. Based upon the $p$-harmonic structure we consider the nonlinear (in the sense of not necessarily linear) Royden harmonic boundary $\Delta_{p}\left(B^{n}\right)$ with exponent $p$ of the ball $B^{n}$ (see $\S 5$ for the precise definition) in addition to the original linear Royden harmonic boundary $\Delta_{2}\left(B^{n}\right)=\Delta\left(B^{n}\right)$ of the ball $B^{n}$. There are many results in the nonlinear potential theory which are superficially formulation-preserving generalizations of those in the linear case. In the present case of $\Delta_{p}\left(B^{n}\right)$, however, certain properties of $\Delta_{p}\left(B^{n}\right)(p \neq 2)$ may differ from those of $\Delta_{2}\left(B^{n}\right)$ in an essential way depending on the exponent $p$, and our main concern of the generalized condenser problem for the ball $B^{n}$ is then settled in the following fashion.

The Main Theorem (Geometric form). The Royden harmonic boundary $\Delta_{p}\left(B^{n}\right)$ with exponent $p(1<p \leqq n)$ of the Euclidean ball $B^{n}$ of dimension $n \geqq 2$ is connected for $2 \leqq p \leqq n$ but disconnected for $1<p<2$.

Corresponding to the above result formulated in a geometric form we restate it in an analytic form. Generalizing the $p$-Laplace equation we consider a quasilinear elliptic equation of the form

$$
-\nabla \cdot \mathscr{A}(x, \nabla u)=0 \quad\left(\mathscr{A} \in \mathscr{A}_{p}\left(B^{n}\right), 1<p \leqq n\right)
$$

on the ball $B^{n}$, the solutions of which are usually called $\mathscr{A}$-harmonic on $B^{n}$. Here $\mathscr{A}_{p}\left(B^{n}\right)$ is the family of all strictly monotone elliptic operators $\mathscr{A}: B^{n} \times R^{n} \rightarrow R^{n}$ with $\mathscr{A}(x, \nabla u) \cdot \nabla u \approx|\nabla u|^{p}$ (see $\S 2$ for the precise assumptions on $\mathscr{A}$ ). Since $|\nabla u|^{p}$ is locally integrable over $B^{n}$ for $\mathscr{A}$-harmonic functions $u$ on $B^{n}\left(\mathscr{A} \in \mathscr{A}_{p}\left(B^{n}\right)\right.$ ), we can define the $p$-Dirichlet integral of $u$ over $B^{n}$ by

$$
D_{p}\left(u ; B^{n}\right)=\int_{B^{n}}|\nabla u(x)|^{p} d x \leqq \infty
$$

and we say that $u$ is $p$-Dirichlet finite on $B^{n}$ if $D_{p}\left(u ; B^{n}\right)<\infty$. We denote by $u \wedge v$ the greatest $\mathscr{A}$-harmonic minorant of two $\mathscr{A}$-harmonic functions $u$ and $v$ on $B^{n}$. An $\mathscr{A}$-harmonic function $w$ on $B^{n}$ is said to be an $\mathscr{A}$-harmonic measure on $B^{n}$ if

$$
w \wedge(1-w)=0
$$

on $B^{n}$. Intuitively speaking, a $p$-Dirichlet finite $\mathscr{A}$-harmonic measure $w$ on $B^{n}$ is the "potential" of the "electric field" of a "condenser" formed by a subdivision of the "ideal boundary" of $B^{n}$. Then our solution to the generalized condenser problem for $B^{n}$ may also be stated in the following fashion. 
The Main Theorem (Analytic form). Every $p$-Dirichlet finite $\mathscr{A}$-harmonic measure on $B^{n}$ reduces to a constant for every $\mathscr{A}_{\text {in }} \mathscr{A}_{p}\left(B^{n}\right)$ with $2 \leqq p \leqq n$; nevertheless, there exist nonconstant $p$-Dirichlet finite $\mathscr{A}$-harmonic measures on $B^{n}$ for every $\mathscr{A}$ in $\mathscr{A}_{p}\left(B^{n}\right)$ with $1<p<2$.

The paper is organized as follows. In $\S 1$ some fundamentals of Sobolev spaces are stated. Especially the comparison of Sobolev null spaces for different exponents $p$ are discussed. The Maz'ja decomposition of the Sobolev space into the class of $\mathscr{A}$-harmonic functions and the null space is considered in $\S 2$. In particular the monotoneity of the decomposition is established. In $\S 3$ basic properties of $\mathscr{A}$-harmonic measures are discussed. The $p$-capacity of the periphery of the $(n-1)$-dimensional ball in the $n$-dimensional Euclidean space is calculated in $\S 4$ only for the sake of completeness since the result is essentially made use of later. As the last of the preparatory sections, the $p$-Royden harmonic boundary is explained in $\$ 5$ and the equivalence of the main theorems in the geometric and the analytic forms is established. After these preliminary discussions in $\S \S 1-5$, the proof of the main theorem is given in $\S 6$ for the analytic form.

\section{Sobolev spaces}

1.1. The length $|x|$ of a point (vector) $x=\left(x^{1}, \ldots, x^{n}\right)$ in the Euclidean space $R^{n}$ of dimension $n \geqq 2$ is given by $\left(\sum_{i=1}^{n}\left(x^{i}\right)^{2}\right)^{1 / 2}$ as usual. We denote by $x \cdot y$ the inner product of $x$ and $y=\left(y^{1}, \ldots, y^{n}\right)$ so that $x \cdot y=\sum_{i=1}^{n} x^{i} y^{i}$ and $x \cdot x$ $=|x|^{2}$. Our main purpose of this paper is to study potential theoretic nature of the open unit ball $B^{n}$ in $R^{n}$ :

$$
B^{n}=\left\{x \in R^{n}:|x|<1\right\} .
$$

We denote by $B(a, r)$ the open ball $\left\{x \in R^{n}|x-a|<r\right\}$ of center $a$ and radius $r$ in $(0, \infty)$. Since we are mainly considering balls $B(0, r)$ with center the origin 0 of $R^{n}$, we simply denote them by $B(r)$ so that $B^{n}=B(1)$ and $R^{n}=B(\infty)$.

Throughout this paper the number $p$ stands for an exponent with $1<p \leqq n$ and $q$ its conjugate exponent so that $1 / p+1 / q=1$. The space $L_{p}(B)$ over a ball $B=B(r)$ is considered with respect to the volume element $d x=d x^{1} \cdots d x^{n}$ on $R^{n}$. The volume (Lebesgue measure) of a measurable set $E \subset R^{n}$ is denoted by $|E|=\int_{E} d x$.

Besides the space $L_{p}(B)$ we consider the product space 


$$
L_{p}(B)^{n}=L_{p}(B) \times \cdots \times L_{p}(B) \quad(n \text { factors }),
$$

which is the space of measurable vector fields $X=\left(X^{1}, \ldots, X^{n}\right): B \rightarrow R^{n}$ with the norm

$$
\left\|X ; L_{p}(B)^{n}\right\|=\left(\int_{B}|X(x)|^{p} d x\right)^{1 / p}<\infty
$$

where $|X|=\left(\sum_{i=1}^{n}\left(X^{i}\right)^{2}\right)^{1 / 2}$ so that $|X| \in L_{p}(B)$ and

$$
\left\||X| ; L_{p}(B)\right\|=\left\|X ; L_{p}(B)^{n}\right\| \text {. }
$$

The space $L_{p}(B)^{n}$ shares the same properties with $L_{p}(B)$. For example, the dual space $\left(L_{p}(B)^{n}\right)^{*}$ of $L_{p}(B)^{n}$ is $L_{q}(B)^{n}(1 / p+1 / q=1)$ in the sense that the mapping $Y^{*} \rightarrow Y$ determined by

$$
Y^{*}(X)=\int_{B} X(x) \cdot Y(x) d x \quad\left(X \in L_{p}(B)\right)
$$

is an isometric isomorphism between $\left(L_{p}(B)^{n}\right)^{*}$ and $L_{q}(B)^{n}$. Hence in particular, $L_{p}(B)^{*}$ is a reflexive Banach space as $L_{p}(B)$ is. The following consequence of the reflexivity is useful: A subset in $L_{p}(B)\left(L_{p}(B)^{n}\right.$, resp.) is sequentially weakly compact if and only if it is bounded in $L_{p}(B)\left(L_{p}(B)^{n}\right.$, resp.) (cf. e.g. [6; p. 68]).

1.2. Recall that a vector field $X \in\left(\operatorname{loc} L_{1}(B)\right)^{n}$, the product of $n$ same factors $\operatorname{loc} L_{1}(B)$, is a distributional gradient $\nabla u=\left(\partial u / \partial x^{1}, \ldots, \partial u / \partial x^{n}\right)$ of a function $u \in \operatorname{loc} L_{1}(B)$ if

$$
\int_{B} u(x) \nabla \cdot Y(x) d x=-\int_{B} X(x) \cdot Y(x) d x
$$

for every vector field $Y \in C_{0}^{\infty}(B)^{n}=C_{0}^{\infty}(B) \times \cdots \times C_{0}^{\infty}(B)$ ( $n$ factors). Our basic function space is the Sobolev space $W_{p}^{1}(B)$ of functions $f \in L_{p}(B)$ whose distributional gradient $\nabla f \in L_{p}(B)^{n}$. It is a Banach space equipped with the norm

$$
\left\|f ; W_{p}^{1}(B)\right\|=\left\|f ; L_{p}(B)\right\|+\left\|\nabla f ; L_{p}(B)^{n}\right\| .
$$

Concerning the smoothness of functions in $W_{p}^{1}(B)$ we know that the subspace $W_{p}^{1}(B) \cap C^{\infty}(B)$ is dense in $W_{p}^{1}(B)$ with respect to the above norm (cf. e.g. [18; p. 12]). The closure of $C_{0}^{\infty}(B)$ in $W_{p}^{1}(B)$ is denoted by $W_{p, 0}^{1}(B)$. Intuitively speaking, a function $f$ in $W_{p}^{1}(B)$ belongs to $W_{p, 0}^{1}(B)$ if and only if $f$ "vanishes" on the "boundary" of $B$. The meaning will be made more precise after we introduce the Royden compactification of $B$ in $\S 5$. 
We can measure the size of the boundary $\partial B$ of $B$ with respect to $W_{p, 0}^{1}(B)$. We say that $B$ is $p$-parabolic (i.e. $\partial B$ is "small") or $p$-hyperbolic (i.e. $\partial B$ is "large") according as $1 \in W_{p, 0}^{1}(B)$ or $1 \notin W_{p, 0}^{1}(B)$. It is seen that $B(\infty)=R^{n}$ is $n$-parabolic (which we do not use in this paper). However we have the following result.

Lemma 1.1. A finite ball $B=B(r)(0<r<\infty)$ is p-hyperbolic, i.e. $1 \notin$ $W_{p, 0}^{1}(B)$.

Proof. Contrariwise suppose that $1 \in W_{p, 0}^{1}(B)$ so that there exists a sequence $\left\{\varphi_{k}\right\}$ in $C_{0}^{\infty}(B)$ such that

$$
\left\|\varphi_{k}-1 ; W_{p}^{1}(B)\right\| \rightarrow 0 \quad(k \rightarrow \infty) .
$$

Let $u(x)=u\left(x^{1}, \ldots, x^{n}\right)=x^{1}$. Since $u$ is bounded on $B$ and $|\nabla u|=1$, we can easily check that

$$
\left\|u \varphi_{k}-u ; W_{p}^{1}(B)\right\| \rightarrow 0 \quad(k \rightarrow \infty) .
$$

The Gauss divergence theorem

$$
\int_{B} \nabla \cdot\left(\left(u \varphi_{k}\right)|\nabla u|^{p-2} \nabla u\right) d x=\int_{\partial B}\left(\left(u \varphi_{k}\right)|\nabla u|^{p-2} \nabla u\right) \cdot \frac{x}{|x|} d S_{x},
$$

where $d S_{x}$ is the area element on $\partial B$, assures that

$$
\int_{B}|\nabla u|^{p-2} \nabla u \cdot \nabla\left(u \varphi_{k}\right) d x=0
$$

since $\varphi_{k}$ vanishes on $\partial B$ and $\nabla \cdot\left(|\nabla u|^{p-2} \nabla u\right)=0$ because of the fact that $|\nabla u|^{p-2} \nabla u=(1,0, \ldots, 0)$. The Hölder inequality shows that the difference of the left hand side of (1.2) and

$$
\int_{B}|\nabla u|^{p-2} \nabla u \cdot \nabla u d x=\int_{B}|\nabla u|^{p} d x
$$

in absolute value is dominated by

$$
\left\||\nabla u|^{p-2} \nabla u ; L_{q}(B)^{n}\right\| \cdot\left\|\nabla u-\nabla\left(u \varphi_{k}\right) ; L_{p}(B)^{n}\right\| \leqq|B|^{1 / q}\left\|u-u \varphi_{k} ; W_{p}^{1}(B)\right\|,
$$

where $1 / p+1 / q=1$. Thus, by (1.1) and (1.2), we must conclude that $\int_{R}|\nabla u|^{p} d x=0$ but on the other hand 


$$
\int_{B}|\nabla u|^{p} d x=\int_{B} d x=|B|>0
$$

We have arrived at a contradiction.

1.3. The following characterization of $W_{p}^{1}(B)$ is a part of the Nikodym theorem (cf. e.g. [16; p. 73], [18; pp. 8-9]): Any $u$ in $W_{p}^{1}(B)$ (possibly modified on a set of zero measure $d x$ ) is absolutely continuous on almost all straight lines which are parallel to coordinate axes. The distributional gradient of $u$ coincides with the usual gradient almost everywhere; Conversely, if a function $u$ in $L_{p}(B)$ is absolutely continuous on almost all straight lines that are parallel to coordinate axes and the usual gradient of $u$ belongs to $L_{p}(B)$, then it coincides with the distributional gradient of $u$ and $u \in W_{p}^{1}(B)$.

Using the above characterization of the class $W_{p}^{1}(B)$, we see the following useful lattice property of $W_{p}^{1}(B)$. For two functions $f_{1}$ and $f_{2}$ on $B$, we define new functions $f_{1} \cup f_{2}$ and $f_{1} \cap f_{2}$ on $B$ by

$$
\left(f_{1} \cup f_{2}\right)(x)=\max \left(f_{1}(x), f_{2}(x)\right),\left(f_{1} \cap f_{2}\right)(x)=\min \left(f_{1}(x), f_{2}(x)\right)
$$

for each point $x$ in $B$.

Concerning these lattice operations $U$ and $\cap$, the Sobolev space $W_{p}^{1}(B)$ forms a vector lattice; The following identities hold:

$$
\begin{gathered}
\left\|f_{1} \cup f_{2} ; L_{p}(B)\right\|^{p}+\left\|f_{1} \cap f_{2} ; L_{p}(B)\right\|^{p}=\left\|f_{1} ; L_{p}(B)\right\|^{p}+\left\|f_{2} ; L_{p}(B)\right\|^{p}, \\
\left\|\nabla\left(f_{1} \cup f_{2}\right) ; L_{p}(B)^{n}\right\|^{p}+\left\|\nabla\left(f_{1} \cap f_{2}\right) ; L_{p}(B)^{n}\right\|^{p} \\
=\left\|\nabla f_{1} ; L_{p}(B)^{n}\right\|^{p}+\left\|\nabla f_{2} ; L_{p}(B)^{n}\right\|^{p} ;
\end{gathered}
$$

The mapping $(f, g) \rightarrow f \cup g, f \cap g$ of $W_{p}^{1}(B) \times W_{p}^{1}(B)$ to $W_{p}^{1}(B)$ is continuous: if $\left\{f_{k}\right\}$ and $\left\{g_{k}\right\}$ are sequences in $W_{p}^{1}(B)$ such that $\left\|f_{k}-f ; W_{p}^{1}(B)\right\| \rightarrow 0$ and $\left\|g_{k}-g ; W_{p}^{1}(B)\right\| \rightarrow 0$ as $k \rightarrow \infty$ for functions $f$ and $g$ in $W_{p}^{1}(B)$, then $\| f_{k} \cup g_{k}$ $-f \cup g ; W_{p}^{1}(B) \| \rightarrow 0$ and $\left\|f_{k} \cap g_{k}-f \cap g ; W_{p}^{1}(B)\right\| \rightarrow 0$ as $k \rightarrow \infty$.

The basic idea of the proofs for these facts can be found e.g. in [4; pp. $69-70$, pp. 78-79], in which the dimension $n$ is restricted to 2 and the function space in consideration is slightly different from the present $W_{p}^{1}(B)$.

1.4. We insert here a proof of the following elementary fact for the sake of completeness. Let $B=B(r)$ be a finite ball and $B_{1}=B\left(r_{1}\right)$ a concentric ball such that $\bar{B} \subset B_{1}$ so that $0<r<r_{1} \leqq \infty$. With each function $f$ on $B_{1}$ we associate the function $f_{t}$ on $B$ for each $t \in\left(0, r_{1} / r\right)$ given pointwise by 


$$
f_{t}(x)=f(t x) \quad\left(|x|<r_{1} / t\right)
$$

Lemma 1.2. If $f$ belongs to $L_{p}\left(B_{1}\right)$, then $f_{t}$ belongs to $L_{p}(B)\left(0<t<r_{1} / r\right)$ and

$$
\lim _{t \rightarrow 1}\left\|f_{t}-f ; L_{p}(B)\right\|=0 .
$$

Proof. Fix a closed ball $A=\bar{B}\left(r_{2}\right)$ with $r<r_{2}<r_{1}$. For an arbitrary number $\varepsilon>0$ there exists a $g$ in $C(A)$ such that

$$
\left\|g-f ; L_{p}(A)\right\|<\varepsilon
$$

(cf. e.g. [27; p. 69]). Clearly $f_{t} \in L_{p}(B)\left(0<t<r_{1} / r\right)$. Observe that

$$
\left\|f_{t}-f ; L_{p}(B)\right\| \leqq\left\|f_{t}-g_{t} ; L_{p}(B)\right\|+\left\|g_{t}-g ; L_{p}(B)\right\|+\left\|g-f ; L_{p}(B)\right\|
$$

for every $t \in\left(0, r_{2} / r\right)$. The first term of the right hand side of the above inequality is, by the change of integrating variable $\zeta=t x$,

$$
\begin{aligned}
& \left(\int_{B}\left|f_{t}(x)-g_{t}(x)\right|^{p} d x\right)^{1 / p}=\left(\int_{B}|f(t x)-g(t x)|^{p} d x\right)^{1 / p} \\
= & \left(\int_{t B}|f(\zeta)-g(\zeta)|^{p} t^{-n} d \zeta\right)^{1 / p} \leqq t^{-n / p}\left\|f-g ; L_{p}(A)\right\| \leqq t^{-n / p} \varepsilon
\end{aligned}
$$

where $t B=B(t r) \subset A\left(t \in\left(0, r_{2} / r\right)\right.$. Therefore we see that

$$
\limsup _{t \rightarrow 1}\left\|f_{t}-f ; L_{p}(B)\right\| \leqq 2 \varepsilon+\lim \sup _{t \rightarrow 1}\left\|g_{t}-g ; L_{p}(B)\right\| .
$$

Since $g \in C(A)$, the uniform continuity of $g$ on $A \supset B$ assures that

$$
\lim _{t \rightarrow 1}\left\|g_{t}-g ; L_{\infty}(B)\right\|=0 \text {. }
$$

In view of the inequality

$$
\left\|g_{t}-g ; L_{p}(B)\right\| \leqq|B|^{1 / p}\left\|g_{t}-g ; L_{\infty}(B)\right\|,
$$

we see that $\lim \sup _{t \rightarrow 1}\left\|g_{t}-g ; L_{p}(B)\right\|=0$ and therefore

$$
\limsup _{t \rightarrow 1}\left\|f_{t}-f ; L_{p}(B)\right\| \leqq 2 \varepsilon \text {. }
$$

On letting $\varepsilon \downarrow 0$ we obtain $\lim _{t \rightarrow 1}\left\|f_{t}-f ; L_{p}(B)\right\|=0$.

Corollary. If $f$ belongs to $L_{p}(B)$, then $f_{t}$ belongs to $L_{p}(B)(0<t<1)$ and 


$$
\lim _{t \uparrow 1}\left\|f_{t}-f ; L_{p}(B)\right\|=0 .
$$

Proof. We extend $f$ to $R^{n}$ by setting $f=0$ on $R^{n} \backslash B$. Then $f \in L_{p}\left(R^{n}\right)$ and Lemma 1.2 implies $\lim _{t \rightarrow 1}\left\|f_{t}-f ; L_{p}(B)\right\|=0$ and in particular the above assertion.

1.5. As in the preceding subsections let $B=B(r)$ be a finite ball and $B_{1}=$ $B\left(r_{1}\right)$ the concentric ball with $\bar{B} \subset B_{1}$. As a consequence of Lemma 1.2 we have the following result.

LEMMA 1.3. If $f$ belongs to $W_{p}^{1}\left(B_{1}\right)$, then $f_{t}$ belongs to $W_{p}^{1}(B)\left(0<t<r_{1} / r\right)$ and

$$
\lim _{t \rightarrow 1}\left\|f_{t}-f ; W_{p}^{1}(B)\right\|=0
$$

Proof. In view of Lemma 1.2 we only have to prove

$$
\lim _{t \rightarrow 1}\left\|\nabla f_{t}-\nabla f ; L_{p}(B)^{n}\right\|=0 .
$$

Observe that

$$
\frac{\partial f_{t}}{\partial x^{i}}(x)=\frac{\partial}{\partial x^{i}}(f(t x))=t \frac{\partial f}{\partial x^{i}}(t x)=t\left(\frac{\partial f}{\partial x^{i}}\right)_{t}(x)
$$

almost everywhere on $B$, i.e. $\partial f_{t} / \partial x^{i}=t\left(\partial f / \partial x^{i}\right)_{t}$, and therefore

$$
\left|\nabla f_{t}-\nabla f\right| \leqq \sum_{i=1}^{n}\left|\frac{\partial f_{t}}{\partial x^{i}}-\frac{\partial f}{\partial x^{i}}\right| \leqq t \sum_{i=1}^{n}\left|\left(\frac{\partial f}{\partial x^{i}}\right)_{t}-\frac{\partial f}{\partial x^{i}}\right|+|1-t| \sum_{i=1}^{n}\left|\frac{\partial f}{\partial x^{i}}\right| .
$$

A fortiori we have

$$
\left\|\nabla f_{t}-\nabla f ; L_{p}(B)^{n}\right\| \leqq t \sum_{i=1}^{n}\left\|\left(\frac{\partial f}{\partial x^{i}}\right)_{t}-\frac{\partial f}{\partial x^{i}} ; L_{p}(B)\right\|+|1-t| \sum_{i=1}^{n}\left\|\frac{\partial f}{\partial x^{i}} ; L_{p}(B)\right\| .
$$

Since $\partial f / \partial x^{i} \in L_{p}(B) \quad(i=1, \ldots, n)$, Lemma 1.2 assures that the first term of the right hand side of the above inequality tends to zero as $t \rightarrow 1$. We can thus conclude (1.3).

1.6. Let $B=B(r)$ be a finite ball $(0<r<\infty)$. It is natural to extend each $\varphi$ in $C_{0}^{\infty}(B)$ to $R^{n}$ by setting $\varphi=0$ on $R^{n} \backslash B$. In this sense we usually view that $C_{0}^{\infty}(B) \subset C^{\infty}\left(R^{n}\right)$ and actually

$$
C_{0}^{\infty}(B)=\left\{f \in C^{\infty}\left(R^{n}\right): \operatorname{supp} f \subset B\right\} .
$$


Imitating this situation we extend each $g$ in $W_{p, 0}^{1}(B)$ to $R^{n}$ by setting $g=0$ on $R^{n} \backslash B$. However, this time, the inclusion $W_{p, 0}^{1}(B) \subset W_{p}^{1}\left(R^{n}\right)$ may not be entirely trivial and all the more for the following assertion a proof may be in order.

Lemma 1.4. Any function $f$ in $W_{p, 0}^{1}(B)$ can be continued to a function in $W_{p}^{1}\left(R^{n}\right)$ by setting $f=0$ on $R^{n} \backslash B$. Conversely any function $f$ in $W_{p}^{1}\left(R^{n}\right)$ vanishing on $R^{n} / B$ belongs to $W_{p, 0}^{1}(B)$. In short,

$$
W_{p, 0}^{1}(B)=\left\{f \in W_{p}^{1}\left(R^{n}\right): f \mid R^{n} \backslash B=0\right\} .
$$

Proof. To be precise we denote by $f^{\sim}$ the extension of any function or any vector field $f$ on $B$ to $R^{n}$ by setting $f^{\sim}=0$ on $R^{n} \backslash B$. We maintain that $g^{\sim}$ belongs to $W_{p}^{1}\left(R^{n}\right)$ for any $g$ in $W_{p, 0}^{1}(B)$. For the purpose choose a sequence $\left\{\varphi_{k}\right\}$ in $C_{0}^{\infty}(B)$ such that $\left\|\varphi_{k}-g ; W_{p}^{1}(B)\right\| \rightarrow 0(k \rightarrow \infty)$. Consider

$$
\int_{R^{n}} g^{\sim}(x) \nabla \cdot Y(x) d x=\int_{B} g(x) \nabla \cdot Y(x) d x
$$

for any vector field $Y$ in $C_{0}^{\infty}\left(R^{n}\right)^{n}$. By the Hölder inequality, we see that

$$
\begin{gathered}
\quad\left|\int_{B} g(x) \nabla \cdot Y(x) d x-\int_{B} \varphi_{k}(x) \nabla \cdot Y(x) d x\right| \\
\leqq\left\|g-\varphi_{k} ; L_{p}(B)\right\| \cdot\left\|\nabla \cdot Y ; L_{q}(B)\right\| \rightarrow 0 \quad(k \rightarrow \infty)
\end{gathered}
$$

where $1 / p+1 / q=1$. Hence

$$
\int_{B} g(x) \nabla \cdot Y(x) d x=\lim _{k \rightarrow \infty} \int_{B} \varphi_{k}(x) \nabla \cdot Y(x) d x .
$$

By the Gauss divergence theorem

$$
\int_{B} \nabla \cdot\left(\varphi_{k}(x) Y(x)\right) d x=\int_{\partial B} \varphi_{k}(x) Y(x) \cdot \frac{x}{|x|} d s_{x}=0
$$

where $d S_{x}$ is the area element on $\partial B$, we obtain

$$
\int_{B} \varphi_{k}(x) \nabla \cdot Y(x) d x=-\int \nabla \varphi_{k}(x) \cdot Y(x) d x .
$$

Once more by the Hölder inequality, we see that

$$
\left|\int_{B} \nabla \varphi_{k}(x) \cdot Y(x) d x-\int_{B} \nabla g(x) \cdot Y(x) d x\right|
$$




$$
\leqq\left\|\nabla \varphi_{k}-\nabla g ; L_{p}(B)^{n}\right\| \cdot\left\|Y ; L_{q}(B)^{n}\right\| \rightarrow 0 \quad(k \rightarrow \infty),
$$

and therefore we deduce

$$
\lim _{k \rightarrow \infty}\left(-\int_{B} \nabla \varphi_{k}(x) \cdot Y(x) d x\right)=-\int_{B} \nabla g(x) \cdot Y(x) d x=-\int_{R^{n}}(\nabla g) \sim(x) \cdot Y(x) d x
$$

We have thus obtained the identity

$$
\int_{R^{n}} g^{\sim}(x) \nabla \cdot Y(x) d x=-\int_{R^{n}}(\nabla g) \sim(x) \cdot Y(x) d x
$$

for every $Y$ in $C_{0}^{\infty}\left(R^{n}\right)^{n}$. This shows that the distributional gradient $\nabla g^{\sim}$ of $g^{\sim}$ is $(\nabla g) \sim$ on $R^{n}$ and

$$
\left\|(\nabla g)^{\sim} ; L_{p}\left(R^{n}\right)^{n}\right\|=\left\|\nabla g ; L_{p}(B)^{n}\right\|<\infty
$$

so that $g^{\sim} \in W_{p}^{1}\left(R^{n}\right)$ and, by definition, $g^{\sim}=0$ on $R^{n} \backslash B$.

Conversely let $g \in W_{p}^{1}\left(R^{n}\right)$ and $g \mid R^{n} \backslash B=0$. Set $t_{k}=1+1 / k$ and $g_{k}=$ $g_{t_{k}}$, i.e. $g_{k}(x)=g\left(t_{k} x\right)$. By Lemma $1.3, g_{k} \in W_{p}^{1}(B)$, and actually $g_{k} \in$ $W_{p}^{1}\left(R^{n}\right)$ and $g_{k}(x)=0$ for any $x$ in $|x|>k r /(k+1)$ and moreover

$$
\lim _{k \rightarrow \infty}\left\|g_{k}-g ; W_{p}^{1}(B)\right\|=0 \text {. }
$$

As usual we take $\alpha_{\varepsilon}(x)=\varepsilon^{-n} \alpha\left(\varepsilon^{-1} x\right) \quad(\varepsilon>0)$ where

$$
\alpha(x)=\left(\int_{B^{n}} e^{-1 /\left(1-|x|^{2}\right)} d x\right)^{-1} e^{-1 /\left(1-|x|^{2}\right)}
$$

for $|x|<1$ and $\alpha(x)=0$ for $|x| \geqq 1$ and we form the regularization (mollifier)

$$
g_{k} * \alpha_{\varepsilon}(x)=\int_{R^{n}} g_{k}(x-\xi) \alpha_{\varepsilon}(\xi) d \xi
$$

for every $\varepsilon$ in $0<\varepsilon<r / 2(k+1)$. Then $g_{k} * \alpha_{\varepsilon} \in C_{0}^{\infty}(B)$ and we can choose $\varepsilon_{k}$ in $0<\varepsilon_{k}<r / 2(k+1)$ so small that

$$
\left\|g_{k} * \alpha_{\varepsilon_{k}}-g_{k} ; W_{p}^{1}(B)\right\|<1 / k \text {. }
$$

Then $\left\|g_{k} * \alpha_{\varepsilon_{k}}-g ; W_{p}^{1}(B)\right\| \rightarrow 0(k \rightarrow \infty)$. This assures that $g \in W_{p, 0}^{1}(B)$.

1. 7. Let $B=B(r)$ be a finite ball $(0<r<\infty)$. We take a pair $(a, b)$ of two exponents such that $1<a \leqq b \leqq n$. By the Hölder inequality we see that

$$
\left\|f ; W_{a}^{1}(B)\right\| \leqq|B|^{1 / a-1 / b}\left\|f ; W_{b}^{1}(B)\right\| \quad(1<a \leqq b \leqq n)
$$


for any $f$ in $\operatorname{loc} L_{1}(B)$ with distributional $\nabla f \in\left(\operatorname{loc} L_{1}(B)\right)^{n}$. As a consequence of the above inequality (1.5) we have the trivial inclusion relation

$$
W_{a}^{1}(B) \supset W_{b}^{1}(B) \quad(1<a \leqq b \leqq n) .
$$

Slightly less trivial is the inclusion

$$
W_{a, 0}^{1}(B) \supset W_{b, 0}^{1}(B) \quad(1<a \leqq b \leqq n) .
$$

In fact, $g \in W_{b, 0}^{1}(B)$ means that there exists a sequence $\left\{\varphi_{k}\right\}$ in $C_{0}^{\infty}(B)$ such that $\left\|\varphi_{k}-g ; W_{b}^{1}(B)\right\| \rightarrow 0(k \rightarrow \infty)$. By $(1.5)$

$$
\left\|\varphi_{k}-g ; W_{a}^{1}(B)\right\| \leqq|B|^{1 / a-1 / b}\left\|\varphi_{k}-g ; W_{b}^{1}(B)\right\|
$$

and therefore $\left\|\varphi_{k}-g ; W_{a}^{1}(B)\right\| \rightarrow 0(k \rightarrow \infty)$ and a fortiori $g \in W_{a, 0}^{1}(B)$.

By the above two inclusion relations we see that

$$
W_{a, 0}^{1}(B) \cap W_{b}^{1}(B) \supset W_{b, 0}^{1}(B) \quad(1<a \leqq b \leqq n),
$$

which is the best conclusion we can make if we only use the property of $B$ that it has a finite volume. Due to the nice geometric property of $B$ that the boundary $\partial B$ of $B$ is compact and smooth we can maintain the following important result:

Lemma 1. 5. If $B$ is a finite ball, then

$$
W_{a, 0}^{1}(B) \cap W_{b}^{1}(B)=W_{b, 0}^{1}(B) \quad(1<a \leqq b \leqq n) .
$$

Proof. We only have to show that the left hand side of (1.6) is contained in the right hand side of (1.6). For the purpose we take an arbitrary $f$ in $W_{a, 0}^{1}(B) \cap$ $W_{b}^{1}(B)$ and denote by $\tilde{f}^{\sim}$ the extension of $f$ to $R^{n}$ by setting $f^{\sim}=0$ on $R^{n} \backslash B$. Since $f \in W_{a, 0}^{1}(B),(1.4)$ and its proof imply that $\tilde{f}^{\sim} \in W_{a}^{1}\left(R^{n}\right)$ and $\nabla f^{\sim}=(\nabla f)^{\sim}$ on $R^{n}$. On the other hand, $f \in W_{b}^{1}(B)$ assures that

$$
\left\|f^{\sim} ; W_{b}^{1}\left(R^{n}\right)\right\|=\left\|f ; W_{b}^{1}(B)\right\|<\infty
$$

and thus $f^{\sim} \in W_{b}^{1}\left(R^{n}\right)$. Again by (1.4) we conclude that $f \in W_{b, 0}^{1}(B)$.

\section{A-harmonic functions}

2.1. We say that $\mathscr{A}$ is a strictly monotone elliptic operator on a ball $B=B(r)(0<r \leqq \infty)$ with exponent $p \in(1, n]$ if $\mathscr{A}$ is a mapping of $B \times R^{n}$ to $R^{n}$ satisfying the following assumptions for some constants $0<\alpha \leqq \beta<\infty$ : 
the function $h \mapsto \mathscr{A}(x, h)$ is continuous for almost every fixed $x \in B$, and the function $x \mapsto \mathscr{A}(x, h)$ is measurable for all fixed $h \in R^{n}$;

for almost every $x \in B$ and for all $h \in R^{n}$

$$
\begin{gathered}
\mathscr{A}(x, h) \cdot h \geqq \alpha|h|^{p}, \\
|\mathscr{A}(x, h)| \leqq \beta|h|^{p-1}, \\
\left(\mathscr{A}\left(x, h_{1}\right)-\mathscr{A}\left(x, h_{2}\right)\right) \cdot\left(h_{1}-h_{2}\right)>0
\end{gathered}
$$

whenever $h_{1} \neq h_{2}$, and

$$
\mathscr{A}(x, \lambda h)=|\lambda|^{p-2} \lambda \mathscr{A}(x, h)
$$

for all $\lambda \in R \backslash\{0\}$.

The class of all operators $\mathscr{A}$ on $B$ satisfying (2.1)-(2.5) with the exponent $p$ in $1<p \leqq n$ will be denoted by $\mathscr{A}_{p}(B)$. Using $\mathscr{A} \in \mathscr{A}_{p}(B)$ we consider a quasilinear elliptic equation

$$
-\nabla \cdot \mathscr{A}(x, \nabla u)=0
$$

on $B$. A function $u$ on $B$ is a weak solution of (2.6) if $u \in \operatorname{loc} W_{p}^{1}(B)$ and

$$
\int_{B} \mathscr{A}(x, \nabla u(x)) \cdot \nabla \varphi(x) d x=0
$$

for every $\varphi \in C_{0}^{\infty}(B)$. If $u \in W_{p}^{1}(B)$, then it is easy to see by the Hölder inequality and $\mathscr{A}(x, \nabla u) \in L_{q}(B)^{n}(1 / p+1 / q=1)$ as a consequence of (2.3) that $u$ is a weak solution of (2.6) if and only if (2.7) is valid for every $\varphi \in W_{p, 0}^{1}(B)$. As well-known, weak solutions of (2.6) (possibly modified on sets of zero measure $d x$ ) are actually continuous and in fact Hölder continuous (cf. e.g. [29], [30]). Hereafter we say that a function $u$ on $B$ is $\mathscr{A}$-harmonic if $u \in C(B) \cap \operatorname{loc} W_{p}^{1}(B)$ and $u$ is a weak solution of (2.6) on $B$. We will denote by

$$
W_{p, \mathscr{A}}^{1}(B)=\left\{u \in W_{p}^{1}(B): u \text { is } \mathscr{A} \text {-harmonic on } B\right\} .
$$

A simple characterization of the class $W_{p, \mathscr{A}}^{1}(B)$ that it is the class of all $p$-Dirichlet finite $\mathscr{A}$-harmonic functions on $B$ will be given in the next subsection as (2.9).

The simplest and the most typical operator $\mathscr{A}$ in $\mathscr{A}_{p}(B)$ is the $p$-Laplacian

$$
\mathscr{A}(x, h)=|h|^{p-2} h
$$


so that the corresponding elliptic equation is the $p$-Laplace equation

$$
-\nabla \cdot\left(|\nabla u|^{p-2} \nabla u\right)=0
$$

whose weak solutions (automatically continuous) are referred to as being $p$-harmonic. Observe that 2-harmonic functions are usual classical harmonic functions. We in particular denote by $W_{p, p}^{1}(B)$ the space of $p$-harmonic functions on $B$ belonging to the class $W_{p}^{1}(B)$, i.e.

$$
W_{p, p}^{1}(B)=W_{p, \mathscr{A}}^{1}(B) \quad\left(\mathscr{A}=|h|^{p-2} h\right) .
$$

Fundamental properties of $\mathscr{A}$-harmonic functions are concisely compiled in e.g. [9] among which we especially use the Harnack inequality (cf. e.g. [26; pp. 295-309]): If $K$ is a closed concentric ball in $B$, then there is a constant $c=$ $c(n, p, \alpha, \beta, K, B)>1$ such that

$$
\sup _{K} u \leqq c \cdot \inf _{K} u
$$

for every nonnegative $\mathscr{A}$-harmonic function $u$ in $B$.

2.2. It is convenient to consider another type of Sobolev space $L_{p}^{1}(B)$, which is the class of functions $f$ in $\operatorname{loc} L_{1}(B)$ whose distributional gradients $\nabla f$ belong to $L_{p}(B)^{n}$. Hence $L_{p}^{1}(B) \cap L_{p}(B)=W_{p}^{1}(B)$. Since the ball $B$ has the cone property (cf. e.g. [18; p. 18]), we have the following generalized Poincare inequality ([18; p. 22]): If $B=B(r)$ is a finite ball $(0<r<\infty)$, then there exists a domain constant $K=K(B)$ such that there exists a constant $c(u)$ with

$$
\left\|u-c(u) ; L_{p}(B)\right\| \leqq K\left\|\nabla u ; L_{p}(B)^{n}\right\| .
$$

Hence we see that

$$
L_{p}^{1}(B)=W_{p}^{1}(B) \quad(B=B(r), 0<r<\infty),
$$

i.e. $L_{p}^{1}(B) \subset L_{p}(B)$.

Recall that the $p$-Dirichlet integral $D_{p}(f ; B)$ of a function $f \in \operatorname{loc} L_{p}^{1}(B)=$ $\operatorname{loc} W_{p}^{1}(B)$ is nothing but the $p^{\text {th }}$ power of the norm $\left\|\nabla f ; L_{p}(B)^{n}\right\|$ :

$$
D_{p}(f ; B)=\int_{B}|\nabla f(x)|^{p} d x \leqq \infty
$$

and $f$ is said to be $p$-Dirichlet finite if $D_{p}(f ; B)<\infty$. We are interested in $p$-Dirichlet finite $\mathscr{A}$-harmonic functions $\left(\mathscr{A} \in \mathscr{A}_{p}(B)\right)$. Concerning these functions we have, as a consequence of (2.8), 


$$
W_{p, \mathscr{A}}^{1}(B)=\left\{u: u \text { is } \mathscr{A} \text {-harmonic on } B \text { and } D_{p}(u ; B)<\infty\right)
$$

for any finite ball $B$ and any $\mathscr{A} \in \mathscr{A}_{p}(B)$.

Still assume that $B=B(r)$ is a finite ball $(0<r<\infty)$. The original Poincaré inequality (cf. e.g. [16; p. 169]) concerns primarily about $\varphi$ in $C_{0}^{\infty}(B)$ :

$$
\left\|\varphi ; L_{p}(B)\right\| \leqq 2 p^{-1 / p} r\left\|\nabla \varphi ; L_{p}(B)^{n}\right\| .
$$

The inequality extends to functions $\varphi$ in $W_{p, 0}^{1}(B)$ by continuity.

2.3. Let $B=B(r)$ be a finite ball $(0<r<\infty)$. For any $f \in W_{p}^{1}(B)$ and $\mathscr{A}$ $\in \mathscr{A}_{p}(B)$ there exists a $p$-Dirichlet finite $\mathscr{A}$-harmonic function $u$ (i.e. $u \in$ $W_{p, A}^{1}(B)$ ) such that $u-f \in W_{p, 0}^{1}(B)$ (cf [17]). Such a function is seen to be unique. In fact, let $u_{i}$ be in $W_{p, \mathscr{A}}^{1}(B)$ with $u_{i}-f \in W_{p, 0}^{1}(B) \quad(i=1,2)$. Since

$$
\int_{B} \mathscr{A}\left(x, \nabla u_{\imath}\right) \cdot \nabla\left(u_{j}-f\right) d x=0 \quad(i, j=1,2),
$$

we have

$$
\int_{B}\left(\mathscr{A}\left(x, \nabla u_{1}\right)-\mathscr{A}\left(x, \nabla u_{2}\right)\right) \cdot\left(\nabla u_{1}-\nabla u_{2}\right) d x=0
$$

The condition (2.4) implies that $\nabla\left(u_{1}-u_{2}\right)=0$ or $u_{1}-u_{2}=c$, a constant, on $B$. In view of the fact that

$$
c=u_{1}-u_{2}=\left(u_{1}-f\right)-\left(u_{2}-f\right) \in W_{p, 0}^{1}(B),
$$

Lemma 1.1 assures that $c=0$ so that $u_{1}=u_{2}$ on $B$.

Since the function $u \in W_{p, \mathcal{A}}^{1}(B)$ with $u-f \in W_{p, 0}^{1}(B)$ is determined uniquely, we denote $u$ by $\pi_{\mathscr{A}}^{B} f$, which will be referred to as the $\mathscr{A}$-harmonic part of $f \in$ $W_{p}^{1}(B)$. We also write the $p$-harmonic part by $\pi_{p}^{B} f=\pi_{\mathscr{A}}^{B} f\left(\mathscr{A}(x, h)=|h|^{p-2} h\right)$. We have thus obtained the direct sum decomposition

$$
W_{p}^{1}(B)=W_{p, \mathscr{A}}^{1}(B) \oplus W_{p, 0}^{1}(B) .
$$

Let $\left\{\boldsymbol{r}_{k}\right\}$ be a strictly increasing sequence in $(0, r)$ converging to $r$ and $B_{k}=$ $B\left(r_{k}\right)(k=1,2, \ldots)$ so that $\left\{B_{k}\right\}$ is an exhaustion of $B$. For any $f \in W_{p}^{1}(B)$, set

$$
u=\pi_{\mathscr{A}}^{B} f
$$

on $B$ and

$$
u_{k}= \begin{cases}\pi_{\mathscr{A}}^{B_{k}} f & \text { on } B_{k}, \\ f & \text { on } B \backslash B_{k} .\end{cases}
$$


We have the following consistency relation (cf. [17]): $\nabla u_{k} \rightarrow \nabla u$ weakly in $L_{p}(B)^{n}$ and $\mathscr{A}\left(x, \nabla u_{k}\right) \rightarrow \mathscr{A}(x, \nabla u)$ weakly in $L_{q}(B)^{n}(1 / p+1 / q=1)$. Further we maintain the following consistency relation.

Lemma 2.1. The sequence $\left\{u_{k}\right\}$ is contained in $W_{p}^{1}(B)$ and converges to $u$ strongly in $L_{p}(B)$ :

$$
\lim _{k \rightarrow \infty}\left\|u_{k}-u ; L_{p}(B)\right\|=0
$$

Proof. Put $g_{k}=f-u_{k}(k-1,2, \ldots)$ and $g=f-u$. Since $g_{k} \in W_{p, 0}^{1}\left(B_{k}\right)$ and $g_{k}=0$ on $B \backslash B_{k}$, (1.4) implies that $g_{k} \in W_{p, 0}^{1}(B)$ so that $u_{k} \in W_{p}^{1}(B)$. Since

$$
\nabla g_{k}=\nabla f-\nabla u_{k} \rightarrow \nabla g=\nabla f-\nabla u
$$

weakly in $L_{p}(B)^{n},\left\{\left\|\nabla g_{k} ; L_{p}(B)^{n}\right\|\right\}$ is bounded (cf. 1.1). By the Poincaré inequality (2.10), $\left\{\left\|g_{k} ; L_{p}(B)\right\|\right\}$ is also bounded and so is $\left\{\left\|g_{k} ; W_{p}^{1}(B)\right\|\right\}$. In view of the Rellich-Kondrachov theorem (cf. e.g. [2; p. 144]), the imbedding

$$
W_{p, 0}^{1}(B) \rightarrow L_{p}(B)
$$

is compact. Therefore, any subsequence of $\left\{g_{k}\right\}$ contains a subsequence $\left\{h_{k}\right\}$ and an $h \in L_{p}(B)$ such that $\left\|h_{k}-h ; L_{p}(B)\right\| \rightarrow 0(k \rightarrow \infty)$. Observe that

$$
\int_{B} \nabla h_{k} \cdot Y d x=-\int_{B} h_{k} \nabla \cdot Y d x \quad(k=1,2, \ldots)
$$

for every $Y \in C_{0}^{\infty}(B)^{n}$. Since $\nabla h_{k} \rightarrow \nabla g$ weakly in $L_{p}(B)^{n}$ and $h_{k} \rightarrow h$ strongly in $L_{p}(B)$, upon letting $k \rightarrow \infty$ in the above displayed identity, we obtain

$$
\int_{B} \nabla g \cdot Y d x=-\int_{B} h \nabla \cdot Y d x
$$

for every $Y \in C_{0}^{\infty}(B)^{n}$. This means that $\nabla g=\nabla h$ and hence $h-g=c$, a constant, on $B$. We now maintain that $c=0$. Contrariwise suppose that $c \neq 0$. Set $w_{k}$ $=c^{-1}\left(h_{k}-g\right)$. Then $w_{k} \in W_{p, 0}^{1}(B), \nabla w_{k} \rightarrow 0$ weakly in $L_{p}(B)^{n}$, and $w_{k} \rightarrow 1$ strongly in $L_{p}(B)$. Take the function $v(x)=v\left(x^{1}, \ldots, x^{n}\right)=x^{1}$ and the unit vector $e_{1}=(1,0, \ldots, 0)$. Since $v \in W_{p, p}^{1}(B)$ and $v w_{k} \in W_{p, 0}^{1}(B)$, we have

$$
\int_{B}|\nabla v|^{p-2} \nabla v \cdot \nabla\left(v w_{k}\right) d x=0
$$

On the other hand, in view of $|\nabla v|^{p-2} \nabla v=e_{1}$ and

$$
|\nabla v|^{p-2} \nabla v \cdot \nabla\left(v w_{k}\right)=\nabla w_{k} \cdot\left(v e_{1}\right)+w_{k}
$$


and $v e_{1} \in L_{q}(B)^{n}(1 / p+1 / q=1)$, we see that

$$
\begin{gathered}
\int_{B}|\nabla v|^{p-2} \nabla v \cdot \nabla\left(v w_{k}\right) d x=\int_{B} \nabla w_{k} \cdot\left(v e_{1}\right) d x+\int_{B} w_{k} d x \\
\rightarrow \int_{B} 0 \cdot\left(v e_{1}\right) d x+\int_{B} 1 d x=|B|>0
\end{gathered}
$$

as $k \rightarrow \infty$, which is clearly a contradiction. Hence $h=g$ on $B$. We have thus seen that any subsequence of $\left\{g_{k}\right\}$ contains a subsequence converging to $g$ strong. ly in $L_{p}(B)$. A fortiori $\left\|g_{k}-g ; L_{p}(B)\right\| \rightarrow 0(k \rightarrow \infty)$ so that $\| u_{k}-u$; $L_{p}(B) \| \rightarrow 0(k \rightarrow \infty)$.

2.4. Let $B=B(r)$ be a finite ball $(0<r<\infty)$. We use the following form of the maximum principle (cf. [17]): If $u$ and $v$ belong to $W_{p, \mathscr{A}}^{1}(B)$ and $(u-v) \cap 0$ $\in W_{p, 0}^{1}(B)$, then $u \geqq v$ on $B$. As a consequence we have the following monotoneity of the operator $\pi_{\mathscr{A}}^{B}: W_{p}^{1}(B) \rightarrow W_{p, \mathscr{A}}^{1}(B)$.

LEMMA 2.2. If $f_{1}$ and $f_{2}$ belong to $W_{p}^{1}(B)$ and $f_{1} \geqq f_{2}$ on $B$, then $\pi_{\mathscr{A}}^{B} f_{1} \geqq \pi_{\mathscr{A}}^{B} f_{2}$ on $B$.

Proof. Put $\pi_{\mathscr{A}}^{B} f_{i}=u_{i}$ and $u_{i}-f_{i}=g_{i} \in W_{p, 0}^{1}(B) \quad(i=1,2)$ so that

$$
u_{1}-u_{2}=\left(f_{1}-f_{2}\right)+\left(g_{1}-g_{2}\right) \text {. }
$$

Choose a sequence $\left\{\varphi_{k}\right\} \subset C_{0}^{\infty}(B)$ such that

$$
\left\|\left(g_{1}-g_{2}\right)-\varphi_{k} ; W_{p}^{1}(B)\right\| \rightarrow 0 \quad(k \rightarrow \infty)
$$

and set $\phi_{k}=\left(f_{1}-f_{2}\right)+\varphi_{k}(k=1,2, \ldots)$. If $t_{k} \in(0, r)$ is chosen close enough to $r$, then we have

$$
\phi_{k} \cap 0=\left(\left(f_{1}-f_{2}\right)+\varphi_{k}\right) \cap 0=\left(f_{1}-f_{2}\right) \cap 0=0
$$

on $\left\{t_{k}<|x|<r\right\}$ and hence $\phi_{k} \cap 0 \in W_{p, 0}^{1}(B)(k=1,2, \ldots)$. Observe that

$$
\left\|\left(u_{1}-u_{2}\right)-\phi_{k} ; W_{p}^{1}(B)\right\|=\left\|\left(g_{1}-g_{2}\right)-\varphi_{k} ; W_{p}^{1}(B)\right\| \rightarrow 0 \quad(k \rightarrow \infty)
$$

and a fortiori, by subsection 1.3 ,

$$
\left\|\left(u_{1}-u_{2}\right) \cap 0-\phi_{k} \cap 0 ; W_{p}^{1}(B)\right\| \rightarrow 0 \quad(k \rightarrow \infty) .
$$

Therefore we can conclude that $\left(u_{1}-u_{2}\right) \cap 0 \in W_{p, 0}^{1}(B)$ along with $\phi_{k} \cap 0 \in$ $W_{p, 0}^{1}(B)(k=1,2, \ldots)$. By the maximum principle stated at the beginning of this subsection, we obtain that $u_{1} \geqq u_{2}$ on $B$. 
2.5. As in subsection 2.4 the ball $B=B(r)$ is supposed to be finite. Take an arbitrary $\mathscr{A}$-harmonic function $h$ on $B$ not necessarily in $W_{p, \mathscr{A}}^{1}(B)$. By the very definition of $\mathscr{A}$-harmonicity that $h \in \operatorname{loc} W_{p}^{1}(B)$, we see that $h \in W_{p}^{1}(B(t))$ for any $t \in(0, r)$. In view of this we have another type of monotoneity of the operator $\pi_{\mathscr{A}}^{B}$.

Lemma 2.3. If $f$ belongs to $W_{p}^{1}(B)$ and $h$ is an arbitrary $\mathscr{A}$-harmonic function on $B$ such that $f \leqq h(f \geqq h$, resp. $)$ on $B$, then $\pi_{\mathscr{A}}^{B} f \leqq h\left(\pi_{\mathscr{A}}^{B} f \geqq h\right.$, resp. $)$ on $B$.

Proof. As in subsection 2.3 take a strictly increasing sequence $\left\{r_{k}\right\}$ in $(0, r)$ converging to $r$ and put $B_{k}=B\left(r_{k}\right)(k=1,2, \ldots)$ so that $\left\{B_{k}\right\}$ is an ex haustion of $B$. Set $u=\pi_{\mathscr{A}}^{B} f$ and

$$
u_{k}= \begin{cases}\pi_{\mathscr{A}}^{B_{k}} f & \text { on } B_{k}, \\ f & \text { on } B \backslash B_{k} .\end{cases}
$$

By Lemma 2.1, we have that $u$ and $u_{k}$ belong to $W_{p}^{1}(B)$ and $\left\|u-u_{k} ; L_{p}(B)\right\|$ $\rightarrow 0(k \rightarrow \infty)$. Hence, by choosing a subsequence if necessary, we can assume that

$$
\lim _{k \rightarrow \infty} u_{k}(x)=u(x)
$$

almost everywhere on $B$. Fix an arbitrary ball $B(t) \quad(0<t<r)$ and choose $k$ so large that $t<r_{k}$. Since $f$ and $h$ belong to $W_{p}^{1}\left(B_{k}\right)$ and $f \leqq h\left(f \geqq h\right.$, resp.) on $B_{k}$, Lemma 2.2 implies that

$$
u_{k}=\pi_{\mathscr{A}}^{B_{k}} f \leqq \pi_{\mathscr{A}}^{B_{k}} h=h \quad\left(u_{k}=\pi_{\mathscr{A}}^{B_{k}} f \geqq \pi_{\mathscr{A}}^{B_{k}} h=h, \quad \text { resp. }\right)
$$

on $B_{k}$ and in particular

$$
u_{k}(x) \leqq h(x) \quad\left(u_{k}(x) \geqq h(x), \text { resp. }\right)
$$

on $B(t)$. On letting $k \rightarrow \infty$, we see that $u(x) \leqq h(x) \quad(u(x) \geqq h(x)$, resp. $)$ almost everywhere on $B(t)$ and hence everywhere on $B(t)$ by the continuity of $\mathscr{A}$-harmonic functions. Since $t$ is arbitrary, we obtain the desired conclusion.

\section{A-harmonic measures}

3.1. Take an $\mathscr{A} \in \mathscr{A}_{p}(B)$ where $B=B(r) \quad(0<r<\infty)$. We denote by $u \wedge v=u \wedge_{\mathscr{A}} v=u \wedge_{\mathscr{A}, B} v$ the greatest $\mathscr{A}$-harmonic minorant of two $\mathscr{A}$-harmonic functions $u$ and $v$ on $B$. Thus $u \wedge v$ is characterized as the $\mathscr{A}$-harmonic function 
$w$ on $B$ with the following two properties. First, $w \leqq u$ and $v$ on $B$. Second, if $h$ is any $\mathscr{A}$-harmonic function on $B$ such that $h \leqq u$ and $v$ on $B$, then $h \leqq w$ on $B$. Needless to say, the greatest $\mathscr{A}$-harmonic minorant of $u$ and $v$ on $B$ may or may not exist and once we use the notation $u \wedge v$, we understand that the existence of the greatest harmonic minorant of $u$ and $v$ on $B$ is assured. We also use the notation $u \vee v$ to indicate $-((-u) \wedge(-v))$.

We say that $w$ is an $\mathscr{A}$-harmonic measure on $B$ if $w$ is $\mathscr{A}$-harmonic on $B$ and satisfies the condition

$$
w \wedge(1-w)=0
$$

on $B$. Observe that $1-w$ is an $\mathscr{A}$-harmonic measure along with $w$ since we have $(1-w) \wedge(1-(1-w))=(1-w) \wedge w=w \wedge(1-w)=0$. The constant functions 1 and 0 are clearly $\mathscr{A}$-harmonic measures and actually these are only constant harmonic measures and any nonconstant $\mathscr{A}$-harmonic measure $w$ satisfies $0<w<1$ on $B$. In fact, from (3.1) it follows that $0 \leqq w \leqq 1$ on $B$ and hence both of $w$ and $1-w$ are nonnegative $\mathscr{A}$-harmonic function on $B$. If $w \equiv c$, a constant, on $B$, then we see that

$$
c \cap(1-c)=w \wedge(1-w)=0,
$$

which shows that $c=0$ or 1 . By the Harnack inequality we see that $w>0$ and $1-w>0$ on $B$ unless $w$ is a constant on $B$.

An intuitive meaning of (3.1) is that the "boundary values" of $w$ on $\partial B$ is a characteristic function of a set $E$ in $\partial B$ and $w(x)$ is the "measure" of $E$ calculated at $x$. If we view $B$ as an electrostatic condenser bounded by two electrodes $\partial B \backslash E$ grounded and $E$ electrostatically charged so as to produce a unit potential difference on $E$, then the potential of the induced electrostatic field on $B$ is $w$ and the total energy of the charge on $E$ is $D_{p}(w ; B)$. Hence we are mainly interested in the existence or nonexistence of nontrivial $p$-Dirichlet finite $\mathscr{A}$-harmonic measures. Such a problem is usually referred to as the generalized condenser problem. The formulation (3.1) of harmonic measures was first introduced by Heins [8] for 2-harmonic functions (cf. also [21], [22], [23]).

3.2. Let $B=B(r)$ be a finite ball $(0<r<\infty)$ and $\mathscr{A} \in \mathscr{A}_{p}(B)$. Although the condition (3.1) is neat, it is not always easy to see whether a given $\mathscr{A}$-harmonic function satisfies (3.1) or not. Hence we try to reformulate (3.1) for $p$-Dirichlet finite $\mathscr{A}$-harmonic functions. 
Lemma 3.1. The following three conditions for a $p$-Dirichlet finite $\mathscr{A}$-harmonic function $w$ on $B$ are equivalent by pairs:

(a) $w \wedge(1-w)=0$ on $B$;

(b) $w(1-w) \in W_{p, 0}^{1}(B)$;

(c) $w^{2} \in W_{p}^{1}(B)$ and $\pi_{\mathscr{A}}^{B} w^{2}=w$.

Proof. First of all we note by (2.9) that $w \in W_{p}^{1}(B)$ (and actually $w \in$ $\left.W_{p, \mathscr{A}}^{1}(B)\right)$. If $w(1-w) \in W_{p, 0}^{1}(B) \subset W_{p}^{1}(B)$, then the identity

$$
w^{2}=w-w(1-w) \quad\left(w \in W_{p, \mathscr{A}}^{1}(B), w(1-w) \in W_{p, 0}^{1}(B)\right)
$$

shows that $w^{2} \in W_{p}^{1}(B)$ and $\pi_{\mathscr{A}}^{B} w^{2}=w$ so that (b) implies (c). Conversely, if $w^{2} \in W_{p}^{1}(B)$ and $\pi_{\mathscr{A}}^{B} w^{2}=w$, then

$$
w(1-w)=-\left(w^{2}-w\right)=-\left(w^{2}-\pi_{\mathscr{A}}^{B} w^{2}\right) \in W_{p, 0}^{1}(B)
$$

so that (c) implies (b). Thus we have seen that (b) and (c) are mutually equivalent.

Suppose (a) is valid so that $0 \leqq w \leqq 1$ on $B$. Since $0 \leqq w(1-w) \leqq w$ and $1-w$ on $B$, we see by the monotoneity of $\pi_{\mathscr{A}}^{\mathrm{B}}$ (Lemma 2.2) that

$$
0=\pi_{\mathscr{A}}^{B} 0 \leqq \pi_{\mathscr{A}}^{B}(w(1-w)) \leqq \pi_{\mathscr{A}}^{B} w=w \text { and } \pi_{\mathscr{A}}^{B}(1-w)=1-w
$$

on $B$. Hence $0 \leqq \pi_{\mathscr{A}}^{B}(w(1-w)) \leqq w \wedge(1-w)=0$ on $B$ and therefore we have $\pi_{\mathscr{A}}^{B}(w(1-w))=0$. By the direct sum decomposition (2.11) we can conclude that $w(1-w) \in W_{p, 0}^{1}(B)$ so that (b) or equivalently (c) is valid.

Finally suppose that (b) or equivalently (c) is valid. We wish to derive (a). By the monotoneity of $\pi_{\mathscr{A}}^{B}$ (Lemma 2.2) we see that $w^{2} \geqq 0$ implies

$$
w=\pi_{\mathscr{A}}^{B} w^{2} \geqq \pi_{\mathscr{A}}^{B} 0=0,
$$

i.e. $w \geqq 0$ on $B$. Since $w^{\prime}=1-w$ is also $p$-Dirichlet finite and $\mathscr{A}$-harmonic on $B$ along with $w$ such that $w^{\prime}\left(1-w^{\prime}\right)=w(1-w) \in W_{p, 0}^{1}(B), w^{\prime}$ also satisfies (b) and (c). Hence by the same reasoning as above we see that $w^{\prime} \geqq 0$ or $w \leqq 1$ on $B$. Thus we have established that $w \geqq 0$ and $1-w \geqq 0$ on $B$. Next take any $\mathscr{A}$-harmonic function $h$ on $B$, not necessarily in $W_{p, \mathscr{A}}^{1}(B)$, such that $h \leqq w$ and $1-w$ on $B$. Then we have

$$
h(1-w) \leqq w(1-w) \text { and } w h \leqq w(1-w)
$$

on $B$. By adding these two inequalities, we have $h \leqq 2 w(1-w)$ on $B$. By the second form of the monotoneity of $\pi_{\mathscr{A}}^{B}$ (Lemma 2.3), we see that 


$$
h \leqq \pi_{\mathscr{A}}^{B}(2 w(1-w))=2 \pi_{\mathscr{A}}^{B}(w(1-w))=0
$$

in view of (b), i.e. $h \leqq 0$ on $B$. This means that $w \wedge(1-w)=0$ so that (a) is derived.

3.3. Concerning the ranges of $\mathscr{A}$-harmonic measures we have the following result.

LEMma 3.2. If $w$ is a nonconstant $p$-Dirichlet finite $\mathscr{A}$-harmonic measure $(\mathscr{A} \in$ $\left.\mathscr{A}_{p}(B)\right)$ on a finite ball $B$, then the range $w(B)$ of $w$ on $B$ is the open interval $(0,1)$ :

$$
\inf _{B} w=0 \quad \text { and } \quad \sup _{B} w=1 .
$$

Proof. Let $\sup _{B} w=a$. Since $0 \leqq w \leqq 1$ on $B$, we have $0 \leqq a \leqq 1$. Then $w^{2} \leqq a w$ on $B$. By the characterization (c) of $p$-Dirichlet finite $\mathscr{A}$-harmonic measures in Lemma 3.1 and the monotoneity of $\pi_{\mathscr{A}}^{B}$ (Lemma 2.2), we see that

$$
w=\pi_{\mathscr{A}}^{B} w^{2} \leqq \pi_{\mathscr{A}}^{B}(a w)=a w
$$

on $B$. Since $w$ is not a constant, $w>0$ and $w \leqq a w$ implies that $a \geqq 1$. With the trivial relation $a \leqq 1$ we conclude that $a=1$, i.e. $\sup _{B} w=1$.

Observe that $1-w$ is also a nonconstant $p$-Dirichlet finite $\mathscr{A}$-harmonic measure along with $w$. Applying the above result to $1-w$, we see that

$$
1=\sup _{B}(1-w)=1-\inf _{B} w
$$

or $\inf _{B} w=0$. Thus we have derived (3.2).

REMARK. The above proof is an amelioration of a standard one in the classical 2-harmonic case. In the classical case it is known that $w^{2}$ is subharmonic and $w$ is the least harmonic majorant of $w^{2}$ when $w$ is a 2-harmonic measure on $B$. Using this fact instead of the operator $\pi_{\mathscr{A}}^{B}$ and its monotoneity, the above lemma 3.2 in the 2-harmonic case can be proven without the assumption of 2-Dirichlet finiteness of $w$. We gave the above proof in order to show how the standard classical method can be generalized to the present $\mathscr{A}$-harmonic case. However we append here another proof of Lemma 3.2 which dose not make use of the assumption of the $p$-Dirichlet finiteness of $w$.

The proof depends upon the monotoneity of $u \wedge v$ in $u$ and $v$. Since $w$ is a nonconstant $\mathscr{A}$-harmonic measure, we at least know that $0<w<1$ on $B$. Set $a=\sup _{B} w$ so that $w \leqq a$ on $B$ and $0<a \leqq 1$. Observe that $1-w \geqq 1-a$ on $B$. Since $0 \leqq 1-a<1$ and $0<w<1$ on $B$, we have 


$$
w \geqq(1-a) w \text { and } 1-a \geqq(1-a) w
$$

on $B$. Hence we see that

$$
0=w \wedge(1-w) \geqq w \wedge(1-a) \geqq\{(1-a) w\} \wedge\{(1-a) w\}=(1-a) w \geqq 0
$$

on $B$ and a fortiori $(1-a) w=0$ on $B$, which implies that $a=1$ or $\sup _{B} w=1$. Considering $1-w$ instead of $w$ in the above argument we see that $\sup _{B}(1-$ $w)=1$ or $\inf _{B} w=0$.

3.4. The following lemma will be made essential use of later in the construction of a nonconstant $p$-Dirichlet finite $\mathscr{A}$-harmonic measure on $B^{n}(\mathscr{A} \in$ $\left.\mathscr{A}_{p}\left(B^{n}\right)\right)$ for $p$ in $(1,2)$.

Lemma 3.3. The $\mathscr{A}$-harmonic part $\pi_{\mathscr{A}}^{B} f$ of an $f$ in $W_{p}^{1}(B)$ with the property

$$
f(1-f) \in W_{p, 0}^{1}(B)
$$

is a $p$-Dirichlet finite $\mathscr{A}$-harmonic measure on $B$.

Proof. We only have to show that $w=\pi_{\mathscr{A}}^{B} f$ is an $\mathscr{A}$-harmonic measure. We have the unique decomposition

$$
f=w+g_{1} \quad\left(g_{1} \in W_{p, 0}^{1}(B)\right) .
$$

We also have the trivial identity

$$
f^{2}=f+g_{2} \quad\left(g_{2}=-f(1-f) \in W_{p, 0}^{1}(B)\right) .
$$

Replacing $f$ by $w+g_{1}$ in the above identity we have

$$
\left(w+g_{1}\right)^{2}=\left(w+g_{1}\right)+g_{2}
$$

and therefore we can write

$$
w^{2}=w+g_{3}
$$

where $g_{3}=g_{1}+g_{2}-\left(2 w+g_{1}\right) g_{1}$ is seen to belong to $W_{p, 0}^{1}(B)$. Thus the above identity is the unique decomposition of $w^{2}$ into the harmonic part $w$ and the part $g_{3}$ in $W_{p, 0}^{1}(B)$ so that

$$
\pi_{\mathscr{A}}^{B} w^{2}=w
$$

which shows, by Lemma 3.1 , that $w$ is an $\mathscr{A}$-harmonic measure on $B$. 


\section{Capacity}

4.1. Since we denote by $B^{n}$ the unit ball $\{|x|<1\}$ in the Euclidean space $R^{n}$ of dimension $n \geqq 2$, the unit sphere $\{|x|=1\}$ in $R^{n}$ is denoted by $\partial B^{n}$. We denote by $\sum^{n}$ the unit sphere in the $(n-1)$-dimensional subspace $\left\{x^{n}=0\right\}$ considered in $R^{n}$ :

$$
\Sigma^{n}=\left(\partial B^{n}\right) \cap\left\{x=\left(x^{1}, \ldots, x^{n}\right) \in R^{n}: x^{n}=0\right\} .
$$

Take the tubular neighborhood

$$
T^{n}(t)=\left\{x \in R^{n}: \operatorname{distance}\left(x, \sum^{n}\right)<t\right\} \quad(0<t<1)
$$

of $\sum^{n}$ in $R^{n}$. Consider the $p$-capacity $\operatorname{cap}_{p}\left(\sum^{n} ; t\right)$ of $\sum^{n}$ relative to the open set $T^{n}(t)$ defined by

$$
\operatorname{cap}_{p}\left(\Sigma^{n} ; t\right)=\inf \int_{R^{n}}|\nabla \varphi(x)|^{p} d x \quad(1<p \leqq n)
$$

where the infimum is taken with respect to $\varphi$ which runs over all functions $\varphi$ in $C_{0}^{\infty}\left(R^{n}\right)$ with supports in $T^{n}(t)$ such that $0 \leqq \varphi \leqq 1$ on $R^{n}$ and $\varphi=1$ on a neighborhood of $\sum^{n}$. Here $C_{0}^{\infty}\left(R^{n}\right)$ may be replaced by $W_{p, 0}^{1}\left(R^{n}\right) \cap C\left(R^{n}\right)$ since $C_{0}^{\infty}\left(R^{n}\right)$ is dense in $W_{p, 0}^{1}\left(R^{n}\right) \cap C\left(R^{n}\right)$ with respect to the convergence given jointly by the norm of $W_{p, 0}^{1}\left(R^{n}\right)$ and the local uniform convergence and moreover $W_{p, 0}^{1}\left(R^{n}\right)$ is closed under lattice operations $U$ and $\cap$ where $f \cup g=\max (f, g)$ and $f \cap g=\min (f, g)$ pointwise (cf. Subsection 1.3).

The value of $\operatorname{cap}_{p}\left(\sum^{n} ; t\right)$ depends upon the choice of $p \in(1, n]$ and $t \in$ $(0,1)$. We give a proof for the sake of completeness to the following fact which will be made use of later in $\S 6$ :

$$
\operatorname{cap}_{p}\left(\sum^{n} ; t\right)=0 \quad(1<p \leqq 2,0<t<1) .
$$

The proof will be given in $4.2-4.4$.

4.2. Since $1<p \leqq 2$, the Hölder inequality implies that

$$
\operatorname{cap}_{p}\left(\sum^{n} ; t\right)^{1 / p} \leqq\left|T^{n}(t)\right|^{1 / p-1 / 2} \operatorname{cap}_{2}\left(\sum^{n} ; t\right)^{1 / 2}
$$

where $\left|T^{n}(t)\right|$ is the volume of $T^{n}(t)$. In view of this we only have to prove (4.2) for the sole $p=2: \operatorname{cap}_{2}\left(\sum^{n} ; t\right)=0 \quad(0<t<1)$.

Let $G\left(x, y ; T^{n}(t)\right)$ be the 2-harmonic Green kernel on $T^{n}(t)$ and $d \mu(y)$ the $(n-2)$-dimensional surface element on $\sum^{n}$. We consider the 2 -harmonic Green potential 


$$
w(x)=\int_{\Sigma^{n}} G\left(x, y ; T^{n}(t)\right) d \mu(y)
$$

of the measure $\mu$ defined on $T^{n}(t)$. The potential $w$ is lower semicontinuous on $T^{n}(t)$ and harmonic on $T^{n}(t) \backslash \sum^{n}$ and has boundary values zero on $\partial T^{n}(t)$. We extend $w$ to $R^{n}$ by setting $w=0$ on $R^{n} \backslash T^{n}(t)$. As for the behavior of $w$ on $\sum^{n}$ we maintain

$$
w(x)=+\infty \quad\left(x \in \Sigma^{n}\right) .
$$

If the dimension $n=2$, then $\sum^{2}$ consists of two points $e=(1,0)$ and $-e$ and $\mu=\delta_{e}+\delta_{-e}$, where $\delta_{a}$ is the Dirac measure with its support at $a$. Thus

$$
w(x)=G\left(x, e ; T^{2}(t)\right)+G\left(x,-e ; T^{2}(t)\right)
$$

and (4.3) is clearly satisfied. Hence we may confine ourselves to the case $n \geqq 3$ for the proof of (4.3). In this case, instead of proving (4.3) directly, we consider the Newtonian potential

$$
v(x)=\int_{\Sigma^{n}} \frac{1}{|x-y|^{n-2}} d \mu(y)
$$

of the measure $\mu$ on $R^{n}$. Since there exists a constant $K$ in $(1, \infty)$ such that

$$
K^{-1} \frac{1}{|x-y|^{n-2}} \leqq G\left(x, y ; T^{n}(t)\right) \quad\left(x, y \in \Sigma^{n}\right),
$$

we see that $K^{-1} v(x) \leqq w(x) \quad\left(x \in \Sigma^{n}\right)$. Therefore, for the proof of (4.3), we only have to show that

$$
v(x)=+\infty \quad\left(x \in \Sigma^{n}\right)
$$

4.3. The proof of (4.4) is only computational. Let $V$ be an arbitrary rotation around $x^{n}$-axis. Since $\mu$ is invariant under $V$, we see that $v(V x)=v(x)$ for any $x$ in $R^{n}$. Hence $v(x)=v\left(e_{1}\right)$ for any $x$ in $\sum^{n}$ where $e_{1}=(1,0, \ldots, 0)$. Hence the proof of (4.4) is reduced to that of $v\left(e_{1}\right)=+\infty$. Since $y$ in $\sum^{n}$ has the form $\left(y^{1}\right.$, $\left.\ldots, y^{n-1}, 0\right)$, we see that

$$
\begin{gathered}
v\left(e_{1}\right)=\int_{\Sigma^{n}} \frac{1}{\left|e_{1}-y\right|^{n-2}} d \mu(y) \\
=\int_{\Sigma^{n}} \frac{1}{\left(\left(y^{1}-1\right)^{2}+\left(y^{2}\right)^{2}+\cdots+\left(y^{n-1}\right)^{2}\right)^{(n-2) / 2}} d \mu(y) .
\end{gathered}
$$


Here $y \in \sum^{n}$ means that $\left(y^{1}\right)^{2}+\left(y^{2}\right)^{2}+\cdots+\left(y^{n-1}\right)^{2}=1$ and thus

$$
\begin{aligned}
& v\left(e_{1}\right)=\int_{\Sigma^{n}} \frac{1}{\left(2\left(1-y^{1}\right)\right)^{(n-2) / 2}} d \mu(y) \\
& =2^{n-1} \int_{\Gamma^{n}} \frac{1}{\left(2\left(1-y^{1}\right)\right)^{(n-2) / 2}} d \mu(y),
\end{aligned}
$$

where $\Gamma^{n}=\left\{x=\left(x^{1}, \ldots, x^{n-1}, 0\right):|x|=1, x^{1} \geqq 0(i=1,2, \ldots, n-1)\right\}$. By the change of variables

$$
\begin{gathered}
y^{1}=\cos \theta^{1} \\
y^{2}=\sin \theta^{1} \cos \theta^{2} \\
\cdots \cdots \cdots \\
y^{n-2}=\sin \theta^{1} \cdots \sin \theta^{n-3} \cos \theta^{n-2} \\
y^{n-1}=\sin \theta^{1} \cdots \sin \theta^{n-3} \cos \theta^{n-2}
\end{gathered}
$$

the integrating domain $\Gamma^{n}$ corresponds to the domain

$$
\Theta^{n}=\left\{\left(\theta^{1}, \ldots, \theta^{n-2}\right): 0 \leqq \theta^{i} \leqq \pi / 2(i=1, \ldots, n-2)\right\}
$$

and therefore $v\left(e_{1}\right)$ is equal to

$$
2^{n-1} \int_{\theta^{n}} \frac{\left(\sin \theta^{1}\right)^{n-3}\left(\sin \theta^{2}\right)^{n-4} \cdots\left(\sin \theta^{n-3}\right)}{\left(2\left(1-\cos \theta^{1}\right)\right)^{(n-2) / 2}} d \theta^{1} d \theta^{2} \cdots d \theta^{n-3} d \theta^{n-2}
$$

which is, by the Fubini theorem,

$$
\begin{aligned}
& 2 \int_{0}^{\pi / 2} \cdots \int_{0}^{\pi / 2}\left(\int_{0}^{\pi / 2} \frac{\sin ^{n-3} \theta^{1}}{\sin ^{n-2} \frac{\theta^{1}}{2}} d \theta^{1}\right) \\
\times & \left(\sin \theta^{2}\right)^{n-4} \cdots\left(\sin \theta^{n-3}\right) d \theta^{2} \cdots d \theta^{n-3} d \theta^{n-2} \\
= & 2^{n-2} \int_{0}^{\pi / 2} \cdots \int_{0}^{\pi / 2}\left(\int_{0}^{\pi / 4} \frac{\sin ^{n-3} \theta \cos ^{n-3} \theta}{\sin ^{n-2} \theta} d \theta\right) \\
\times & \left(\sin \theta^{2}\right)^{n-4} \cdots\left(\sin \theta^{n-3}\right) d \theta^{2} \cdots d \theta^{n-3} d \theta^{n-2} .
\end{aligned}
$$

Observe that

$$
\int_{0}^{\pi / 4} \frac{\sin ^{n-3} \theta \cos ^{n-3} \theta}{\sin ^{n-2} \theta} d \theta \geqq(1 / \sqrt{2})^{n-3} \int_{0}^{\pi / 4} \frac{1}{\sin \theta} d \theta \geqq 2^{-(n-3) / 2} \int_{0}^{\pi / 4} \frac{1}{\theta} d \theta=+\infty
$$


and a fortiori $v\left(e_{1}\right)=+\infty$. We have thus completed the proof of (4.3).

4.4. By the Sard theorem (cf. e.g. [15; p.16]) we can find an increasing sequence $\left\{\lambda_{k}\right\}$ of positive numbers $\lambda_{k}$ divergent to $+\infty$ such that each $\lambda_{k}$ is noncritical value of $w(k=1,2, \ldots)$. Observe that, by the Gauss divergence theorem and $\nabla \cdot \nabla w=\Delta w=0$

$$
\begin{gathered}
\int_{T^{n}(t)}\left|\nabla\left(w \cap \lambda_{k}\right)(x)\right|^{2} d x=\int_{\left\{0<w<\lambda_{k}\right\}} \nabla w \cdot \nabla w d x=\int_{\left\{0<w<\lambda_{k}\right\}} \nabla \cdot(w \nabla w) d x \\
=\int_{\partial\left\{0<w<\lambda_{k}\right\}} w \nabla w \cdot \nu d S=\int_{\left\{w=\lambda_{k}\right\}} w \nabla w \cdot \nu d S \\
=\lambda_{k} \int_{\left\{w=\lambda_{k}\right\}} \nabla w \cdot \nu d S=C \lambda_{k}
\end{gathered}
$$

where $\nu$ is the outer unit normal and $d S$ is the area element on $\partial\{0<w$ $\left.<\lambda_{k}\right\}$ and $C=\int_{\left\{w=\lambda_{k}\right\}} \nabla w \cdot \nu d S=\int_{\{w=0\}} \nabla w \cdot \nu d S$ is a constant independent of $k$ which is usually referred to as the flux of $w$ across $\left\{w=\lambda_{k}\right\}$ and also $\{w=0\}$. The function

$$
f_{k}=\left(w \cap \lambda_{k}\right) / \lambda_{k}
$$

belongs to $W_{2,0}^{1}\left(R^{n}\right) \cap C\left(R^{n}\right)$ and actually a competing function for (4.1) with $p=2(k=1,2, \ldots)$. Therefore we see that

$$
\begin{aligned}
\operatorname{cap}_{2}\left(\sum^{n} ; t\right) \leqq & \int_{R^{n}}\left|\nabla f_{k}(x)\right|^{2} d x=\lambda_{k}^{-2} \int_{T^{n}(t)}\left|\nabla\left(w \cap \lambda_{k}\right)(x)\right|^{2} d x \\
& =\lambda_{k}^{-2} \cdot C \lambda_{k}=C / \lambda_{k} \rightarrow 0 \quad(k \rightarrow \infty),
\end{aligned}
$$

which proves (4.2) for $p=2$.

4.5. As an application of (4.2) we obtain the following result.

LEMmA 4.1. There exists a strictly decreasing sequence $\left\{t_{k}\right\}$ in $(0,1)$ convergent to zero and a sequence $\left\{\chi_{k}\right\}$ in $C^{\infty}\left(R^{n}\right)$ such that $0 \leqq \chi_{k} \leqq 1$ on $R^{n}, \chi_{k}=0$ on $T^{n}\left(t_{k+1}\right), \chi_{k}=1$ on $R^{n} \backslash T^{n}\left(t_{k}\right)$, and $D_{p}\left(\chi_{k} ; R^{n}\right)<2^{-k}$ for every integer $k \geqq 1$.

Proof. First choose an arbitrary but then fixed $t_{1}$ in $(0,1)$. Since $\operatorname{cap}_{p}\left(\sum^{n}\right.$; $\left.t_{1}\right)=0$, there exists a $\varphi_{1}$ in $C_{0}^{\infty}\left(R^{n}\right)$ such that $0 \leqq \varphi_{1} \leqq 1$ on $R^{n}$, the support of $\varphi_{1}$ is contained in $T^{n}\left(T_{1}\right), \varphi_{1}=1$ in a neighborhood of $\sum^{n}$ and $D_{p}\left(\varphi_{1}\right.$; 
$\left.R^{n}\right)<2^{-1}$. We set $\chi_{1}=1-\varphi_{1}$ and choose $t_{2}$ in $\left(0, t_{1} / 2\right)$ such that $\chi_{1}=0$ on $T^{n}\left(t_{2}\right)$. After we have chosen $\chi_{1}, \ldots, \chi_{k}$ and $t_{1}, \ldots, t_{k+1}$ as reuired, we take $\varphi_{k+1}$ in $C_{0}^{\infty}\left(R^{n}\right)$ such that $0 \leqq \varphi_{k+1} \leqq 1$ on $R^{n}$, the support of $\varphi_{k+1}$ is contained in $T^{n}\left(t_{k+1}\right), \varphi_{k+1}=1$ in a neighborhood of $\sum^{n}$, and $D_{p}\left(\varphi_{k+1} ; R^{n}\right)<2^{-(k+1)}$. Such a $\varphi_{k+1}$ certainly exists by virtue of the fact that $\operatorname{cap}_{p}\left(\Sigma^{n} ; t_{k+1}\right)=0$. We then set $\chi_{k+1}=1-\varphi_{k+1}$ and choose a $t_{k+2}$ in $\left(0, t_{k+1} / 2\right)$ such that $\chi_{k+1}=0$ on $T^{n}\left(t_{k+1}\right)$. By the mathematical induction, the construction of the required $\left\{t_{k}\right\}$ and $\left\{\varphi_{k}\right\}$ is thus complete.

\section{Royden harmonic boundaries}

5.1. Let $B=B(r)$ be a finite ball $(0<r<\infty)$. We denote by $M_{p}(B)$ the class of bounded continuous functions $f$ on $B$ with distributional gradients $\nabla f$ in $L_{p}(B)^{n}$. Thus in the present case of $B$ we have

$$
M_{p}(B)=W_{p}^{1}(B) \cap L_{\infty}(B) \cap C(B) \quad(1<p \leqq n) .
$$

The class $M_{p}(B)$ forms a commutative Banach algebra equipped with the norm

$$
\left\|f ; M_{p}(B)\right\|=\left\|f ; L_{\infty}(B)\right\|+\left\|\nabla f ; L_{p}(B)^{n}\right\|
$$

which is referred to as the Royden algebra with exponent $p$ or simply $p$-Royden algebra over $B$

An important subalgebra $M_{p, 0}(B)$ of $M_{p}(B)$ is defined as the totality of $f \in$ $M_{p}(B)$ such that there exists a sequence $\left\{\varphi_{k}\right\}$ in $C_{0}^{\infty}(B)$ with the following three conditions: $\left\|\nabla \varphi_{k}-\nabla f ; L_{p}(B)^{n}\right\| \rightarrow 0(k \rightarrow \infty) ; \sup _{k}\left\|\varphi_{k} ; L_{\infty}(B)\right\|<\infty ;\left\{\varphi_{k}\right\}$ converges to $f$ locally uniformly on $B$. The class $M_{p, 0}(B)$ is not only a subalgebra of $M_{p}(B)$ but also an ideal of $M_{p}(B)$ and is called the $p$-Royden potential subalgeb$r a$ of $M_{p}(B)$. In the present case of $B$ we have, as a counterpart of (5.1), the following expression of $M_{p, 0}(B)$ :

$$
M_{p, 0}(B)=W_{p, 0}^{1}(B) \cap L_{\infty}(B) \cap C(B) .
$$

For a proof for the above expression take an arbitrary $f \in M_{p, 0}(B)$ and its associated sequence $\left\{\varphi_{k}\right\} \subset C_{0}^{\infty}(B)$. The condition $\left\|\nabla \varphi_{k}-\nabla f ; L_{p}(B)^{n}\right\| \rightarrow 0$ $(k \rightarrow \infty)$ in particular assures that $\left\{\nabla \varphi_{k}\right\}$ is a Cauchy sequence in $L_{p}(B)^{n}$. Then, by the Poincare inequality, $\left\{\varphi_{k}\right\}$ forms a Cauchy sequence in $L_{p}(B)$ and therefore $\left\{\varphi_{k}\right\}$ forms a Cauchy sequence in $W_{p}^{1}(B)$. Hence there exists a $g \in W_{p}^{1}(B)$ such that $\left\|\varphi_{k}-g ; W_{p}^{1}(B)\right\| \rightarrow 0(k \rightarrow \infty)$. Since $\left\|\varphi_{k}-g ; L_{p}(B)\right\| \rightarrow 0(k \rightarrow \infty)$, a suitable subsequence of $\left\{\varphi_{k}\right\}$ converges to $g$ almost everywhere on $B$. However, since $\left\{\varphi_{k}\right\}$ converges to $f$ locally uniformly on $B$, we see that $f=g \in W_{p, 0}^{1}(B)$ so 
that in particular $f$ belongs to the right hand side of (5.2).

Conversely choose an arbitrary $f \in W_{p, 0}^{1}(B) \cap L_{\infty}(B) \cap C(B)$. We extend $f$ to $R^{n}$ by setting $f=0$ on $R^{n} \backslash B$. Then, by Lemma 1.4 , we see that $f \in$ $W_{p}^{1}\left(R^{n}\right) \cap L_{\infty}\left(R^{n}\right) \cap C\left(R^{n} \backslash \partial B\right)$. First, consider $f_{t}(1<t<\infty)$ defined in 1.4 and observe that, by Lemma $1.3,\left\|\nabla f_{t}-\nabla f ; L_{p}(B)^{n}\right\| \rightarrow 0(t \downarrow 1)$ and, obviously, $\sup _{t>1}\left\|f_{t} ; L_{\infty}\left(R^{n}\right)\right\| \leqq\left\|f ; L_{\infty}(B)\right\|$ and $f_{t} \rightarrow f$ locally uniformly on $B$ by the uniform continuity of $f$ on each compact subset of $B$. Next, let $f_{t} * \alpha_{\varepsilon} \in$ $C_{0}^{\infty}\left(R^{n}\right) \quad(\varepsilon>0)$ be the regularization of $f_{t}$ as in 1.6. Since $\nabla\left(f_{t} * \alpha_{\varepsilon}\right)=\left(\nabla f_{t}\right) *$ $\alpha_{\varepsilon}$, we see that $\left\|\nabla\left(f_{t} * \alpha_{\varepsilon}\right)-\nabla f_{t} ; L_{p}(B)\right\| \rightarrow 0(\varepsilon \downarrow 0)$. We also see that $f_{t} * \alpha_{\varepsilon}$ $\rightarrow f_{t}$ locally uniformly on $B(1 / t)$ since $f_{t} \in C(B(1 / t))$. Clearly $\sup _{\varepsilon>0} \| f_{t} * \alpha_{\varepsilon}$; $L_{\infty}(B)\|\leqq\| f_{t} ; L_{\infty}(B)\|\leqq\| f ; L_{\infty}(B) \|$. Finally, by choosing $t_{k} \downarrow 1$ and $\varepsilon_{k} \downarrow 0$ suitably and by setting $\varphi_{k}=f_{t_{k}} * \alpha_{\varepsilon_{k}}$, we see that $\left\{\varphi_{k}\right\}$ is a required sequence for $f$ to ensure $f \in M_{p, 0}(B)$. The proof of (5.2) is herewith complete.

5.2. The maximal ideal space $B_{p}^{*}$ of $M_{p}(B)$ is a compact Hausdorff space which we call the Royden compactification of $B$ with exponent $p$ or simply $p$-Royden compactification of $B$ since we see that the space $B$ with its original topology is an open and dense subspace of $B_{p}^{*}$. Functions in $M_{p}(B)$ are uniquely continued to $B_{p}^{*}$ so as to be continuous on $B_{p}^{*}$, and in this sense, by the Weierstrass approximation theorem, $M_{p}(B)$ is a dense subspace of $C\left(B_{p}^{*}\right)$ with respect to the supremum norm on $B_{p}^{*}$. We single out the important part of the Royden boundary $B_{p}^{*} \backslash B$ as follows:

$$
\Delta_{p}(B)=\left\{\zeta \in B_{p}^{*}: f(\zeta)=0 \text { for every } f \in M_{p, 0}(B)\right\}
$$

which is referred to as the Royden harmonic boundary with exponent $p$ or simply p-Royden harmonic boundary.

The Euler-Lagrange equation of the variation of $\int_{B}|\nabla f(x)|^{p} d x$ is the nonlinear equation ( $p$-Laplace equation)

$$
-\nabla \cdot\left(|\nabla u|^{p-2} \nabla u\right)=0
$$

which reduces to the usual Laplace equation

$$
-\Delta u=\nabla \cdot \nabla u=0
$$

for $p=2$. For this reason $M_{2}(B)$ is sometimes called the linear Royden algebra whereas $M_{p}(B)$ for general $p \in(1, n]$ the nonlinear Royden algebra in the sense that it is not necessarily linear. Concerning the linear Royden compactifications, see e.g. [4; pp. 96-109], [28; pp. 145-221], [19], [20], [7], etc. among many others 
and for the nonlinear Royden compactifications, see e.g. [14], [13], [24], [32], [31], etc.

5.3. The following relation dual to (5.3) is important in treating the Royden compactification, to which we give a proof different from many others known in literature for the sake of completeness.

Lemma 5.1 (Duality). A function $f$ in $M_{p}(B)$ belongs to $M_{p, 0}(B)$ if and only if $f$ vanishes on $\Delta_{p}(B)$ :

$$
M_{p, 0}(B)=\left\{f \in M_{p}(B): f \mid \Delta_{p}(B)=0\right\} .
$$

Proof. That the left hand side of (5.4) is contained in the right hand side of (5.4) is nothing but the definition (5.3) of $\Delta_{p}(B)$. Thus we only have to show that $f \in M_{p, 0}(B)$ if $f \in M_{p}(B)$ satisfies $f \mid \Delta_{p}(B)=0$. Set $c=\max \{|f(\zeta)|: \zeta \in$ $\left.B_{p}^{*}\right\}$ and choose an arbitrary $\varepsilon>0$. Consider the open subset $U=\left\{\zeta \in B_{p}^{*}\right.$ : $|f(\zeta)|<\varepsilon\}$ which is a neighborhood of $\Delta_{p}(B)$. Since any $\zeta \in R^{*} \backslash U$ is not the common zeros of functions in $M_{p, 0}(B)$, there exists an $f_{\zeta} \in M_{p, 0}(B)$ such that $f_{\zeta}(\zeta) \neq 0$. It is easy to see that $M_{p, 0}(B)$ also forms a vector lattice with lattice operations $U$ and $\cap$ along with $W_{1, p}(B)$ (cf. 1.3). Hence upon replacing $f_{\zeta}$ by $\left(\left(2 / f_{\zeta}(\zeta)\right) f_{\zeta}\right) \cup 0$, we can assume that we could choose $f_{\zeta} \in M_{p, 0}(B)$ such that $f_{\zeta}(\zeta)=2$ and $f_{\zeta} \geqq 0$ on $B_{p}^{*}$. Observe that $V_{\zeta}=\left\{\eta \in B_{p}^{*}: f_{\zeta}(\eta)>1\right\}$ is an open neighborhood of $\zeta$ so that

$$
\underset{\zeta \in B_{p}^{*} \backslash U}{\cup} V_{\zeta} \supset B_{p}^{*} \backslash U .
$$

By the compactness of $B_{p}^{*} \backslash U$, there exists a finite set of points $\zeta_{1}, \ldots, \zeta_{m}$ in $B_{p}^{*} \backslash U$ such that

$$
\bigcup_{j=1}^{m} V_{\zeta_{j}} \supset B_{p}^{*} \backslash U
$$

Then consider the function

$$
g=\left(\sum_{j=1}^{m} f_{\zeta_{j}}\right) \cap 1
$$

on $B_{p}^{*}$. First of all, as above, it belongs to $M_{p, 0}(B)$. Clearly $0 \leqq g \leqq 1$ on $B_{p}^{*}$ and $g=1$ on $B_{p}^{*} \backslash U$. Hence we have the inequality

$$
-\varepsilon-c g<f<\varepsilon+c g
$$


on $B_{p}^{*}$ and in particular on $B$. Observe that

$$
\pi_{\mathscr{A}}^{B}( \pm(\varepsilon+c g))= \pm\left(\varepsilon+c \pi_{\mathscr{A}}^{B} g\right)= \pm \varepsilon
$$

on $B$ since $g \in M_{p, 0}(B) \subset W_{p, 0}^{1}(B)$. By the monotoneity of $\pi_{\mathscr{A}}^{B}$ (Lemma 2.2) we have

$$
-\varepsilon=\pi_{\mathscr{A}}^{B}(-\varepsilon-c g) \leqq \pi_{\mathscr{A}}^{B} f \leqq \pi_{\mathscr{A}}^{B}(\varepsilon+c g)=\varepsilon
$$

on $B$. On letting $\varepsilon \downarrow 0$ we obtain that $\pi_{\mathscr{A}}^{B} f=0$. By the direct sum decomposition (2.11) we see that $f \in W_{p, 0}^{1}(B)$. By (5.2) and $f \in M_{p}(B)$, we conclude that $f \in$ $M_{p, 0}(B)$.

5.4. Corresponding to the class $W_{p, \mathscr{A}}^{1}(B) \quad\left(\mathscr{A} \in \mathscr{A}_{p}(B)\right)$ introduced in 2.1 we now consider the subspace $M_{p, \mathscr{A}}(B)$ of $M_{p}(B)$ given by

$$
M_{p, \mathscr{A}}(B)=\left\{u \in M_{p}(B): u \text { is } \mathscr{A} \text {-harmonic on } B\right\} .
$$

Hence $u \in M_{p, s}(B)$ if and only if $u$ is $p$-Dirichlet finite, bounded, and $\mathscr{A}$-harmonic on $B$; or $u \in M_{p, \mathscr{A}}(B)$ if and only if $u \in W_{p, \mathscr{A}}(B)$ and $\| u$; $L_{\infty}(B) \|<\infty$. Corresponding to the direct sum decomposition (2.11), we have the so-called Royden decomposition of $M_{p}(B)$ :

$$
M_{p}(B)=M_{p, \mathscr{A}}(B) \oplus M_{p, 0}(B) .
$$

For a proof, take an arbitrary $f \in M_{p}(B)$. By (2.11) we have

$$
f=u+g \quad\left(u \in W_{p, \mathscr{A}}^{1}(B), g \in W_{p, 0}^{1}(B)\right) .
$$

By the monotoneity of $\pi_{\mathscr{A}}^{B}$ (Lemma 2.3) we see that $u=\pi_{\mathscr{A}}^{B} f$ is bounded on $B$ and so is $g$. Since $u$ is continuous, the continuity of $f$ implies that of $g$. Thus $f=u+g$ is a decomposition of $f$ in (5.5). The unicity of the decomposition follows from that of (2.11). This completes the proof of (5.5).

The Royden harmonic boundary $\Delta_{p}(B)$ of $B$ plays important roles in the Dirichlet problem for $\mathscr{A}$-harmonic functions. That it is an essential boundary for functions in $M_{p, \mathscr{A}}(B)$ is seen by the following maximum principle (comparison principle): If $u_{1}$ and $u_{2}$ are in $M_{p, \mathscr{A}}(B)$ and satisfy $u_{1} \geqq u_{2}$ on $\Delta_{p}(B)$, then $u_{1} \geqq u_{2}$ on $B$. Hence in particular, any $u \in M_{p, \mathscr{A}}(B)$ is uniquely determined by $u \mid \Delta_{p}(B)$. For example, a $u \in M_{p, \mathscr{A}}(B)$ is constant on $B$ if and only if $u$ is constant on $\Delta_{p, \mathscr{A}}(B)$.

For a proof of the above maximum principle, note that $\left(u_{1}-u_{2}\right) \cap 0 \in$ $M_{p, 0}(B) \subset W_{p, 0}^{1}(B)$. Hence by the maximum principle quoted in 2.4 , we conclude that $u_{1} \geqq u_{2}$ on $B$. 
5.5. We mentioned in 3.1 that, intuitively speaking, an $\mathscr{A}$-harmonic measure on $B$ is an $\mathscr{A}$-harmonic function whose boundary values is a characteristic function on the boundary of $B$. This is precisely realized for $p$-Dirichlet finite $\mathscr{A}$-harmonic measures $\left(\mathscr{A} \in \mathscr{A}_{p}(B)\right)$ on the $p$-Royden harmonic boundary. As we have been assuming in this section, let $B=B(r)$ be a finite ball $(0<r$ $<\infty)$ and $\mathscr{A}$ be any elliptic operator with $\mathscr{A} \in \mathscr{A}_{p}(B)$. Any $p$-Dirichlet finite $\mathscr{A}$-harmonic measure $w$ is continuous on $B$ and $0 \leqq w \leqq 1$ on $B$ and hence $w \in$ $M_{p, \mathscr{A}}(B)$ so that $w$ is continuous on $B_{p}^{*}$.

Lemma 5.2. For any $p$-Dirichlet finite $\mathscr{A}$-harmonic measure $w$ on $B$, there exists an open and compact subset $K$ of the compact space $\Delta_{p}(B)$ such that $w=\chi_{K}$ on $\Delta_{p}(B)$ where $\chi_{K}$ is the characteristic function of $K$ on $\Delta_{p}(B)$, Conversely, for any open and compact subset $K$ of the compact space $\Delta_{p}(B)$, there exists a $p$-Dirichlet finite $\mathscr{A}$-harmonic measure $w$ on $B$ such that $w=\chi_{K}$ on $\Delta_{p}(B)$.

Proof. Let $w$ be a $p$-Dirichlet finite $\mathscr{A}$-harmonic measure on $B$. By Lemma 3.1 we have $w(1-w) \in W_{p, 0}^{1}(B)$ so that $w(1-w) \in M_{p, 0}(B)$. Thus $w(1-w)$ $=0$ on $\Delta_{p}(B)$ and therefore $w$ takes only two values 0 and 1 on $\Delta_{p}(B)$. Put

$$
K=\left\{\zeta \in \Delta_{p}(B): w(\zeta)=1\right\} .
$$

Since $K=\Delta_{p}(B) \cap w^{-1}(1)$, it is a compact subset of $\Delta_{p}(B)$. On the other hand, by the fact that

$$
K=\left\{\zeta \in \Delta_{p}(B): w(\zeta)>0\right\},
$$

it is an open subset of the space $\Delta_{p}(B)$. Thus $K$ is compact and open in $\Delta_{p}(B)$ and $w=\chi_{K}$ on $\Delta_{p}(B)$.

Conversely, let $K$ be any compact and open subset of $\Delta_{p}(B)$. Then $\left(3 \chi_{K}-\right.$ 1) $\in C\left(\Delta_{p}(B)\right)$. By the Urysohn theorem we can find a $\varphi \in C\left(B_{p}^{*}\right)$ such that $\varphi=3 \chi_{K}-1$ on $\Delta_{p}(B)$. Since $M_{p}(B)$ is dense in $C\left(B_{p}^{*}\right)$ with respect to the supremum norm on $B_{p}^{*}$, we can find a $g \in M_{p}(B)$ such that $|g-\varphi|<1$ on $B_{p}^{*}$. Finally let

$$
f=(g \cup 0) \cap 1,
$$

which is in $M_{p}(B)$ by the lattice property of $M_{p}(B)$. By the construction, we see that $f=\chi_{K}$ on $\Delta_{p}(B)$. Then clearly $f(1-f)=0$ on $\Delta_{p}(B)$ and therefore, by Lemma 3.3,

$$
w=\pi_{\mathscr{A}}^{B} f
$$


is a $p$-Dirichlet finite $\mathscr{A}$-harmonic measure on $B$, and $w=f=\chi_{K}$ on $\Delta_{p}(B)$.

5.6. The equivalence of our main theorems in geometric and analytic forms can now be concluded as a result of the above lemma 5.2 .

LEMma 5.3. The following three conditions are equivalent by pairs:

(a) The p-Royden harmonic boundary $\Delta_{p}(B)$ is connected;

(b) There exist no nonconstant $p$-Dirichlet finite $\mathscr{A}$-harmonic measures on $B$ for every $\mathscr{A} \in \mathscr{A}_{p}(B)$;

(c) There exist no nonconstant $p$-Dirichlet finite $\mathscr{A}$-harmonic measures on $B$ for some $\mathscr{A} \in \mathscr{A}_{p}(B)$.

Proof. The condition (b) trivially implies (c). Next, suppose (c) is valid and, contrariwise assume that $\Delta_{p}(B)$ is not connected. Then there exists an open and closed subset $K$ of the compact space $\Delta_{p}(B)$ such that $K \neq \emptyset$ and $\Delta_{p}(B) \backslash K \neq$ $\emptyset$. By Lemma 5.2 , there exists a $p$-Dirichlet finite $\mathscr{A}$-harmonic measure $w$ with $w=\chi_{K}$ on $\Delta_{p}(B)$. Since $w=\chi_{K}$ is not constant on $\Delta_{p}(B), w$ is not constant on $B$, which contradicts (c). Thus we have seen that (c) implies (a). Finally suppose (a) is valid and take any $\mathscr{A} \in \mathscr{A}_{p}(B)$ and any $p$-Dirichlet finite $\mathscr{A}$-harmonic measure $w$ on $B$. We wish to show that $w$ is constant on $B$. Contrariwise, suppose $w$ is not constant on $B$. Again by Lemma 5.2 , there exists an open and compact subset $K$ of $\Delta_{p}(B)$ such that $w=\chi_{K}$ on $\Delta_{p}(B)$. Since $w$ is not constant on $B, w=\chi_{K}$ is not constant on $\Delta_{p}(B)$ so that $K \neq \emptyset$ and $\Delta_{p}(B) \backslash K \neq \emptyset$. Thus $K \cup\left(\Delta_{p}(B) \backslash K\right)$ is a disjoint decomposition of $\Delta_{p}(B)$ into two nonempty mutually disjoint compact sets and thus $\Delta_{p}(B)$ cannot be connected, which contradicts (a).

COROLLARY. The main theorem in geometric form and that in analytic form are equivalent.

\section{Proof of the main theorem}

6.1. In this final section we prove the main theorem in the analytic form. By Corollary to Lemma 5.3, the proof of the main theorem in the geometric form will then also be complete. Recall that $B^{n}=B(0,1)=B(1)=\left\{x \in R^{n}:|x|<1\right\}$ is the open unit ball in the Euclidean space $R^{n}$ of dimension $n \geqq 2$.

In the former (latter, resp.) half, subsections 6.1-6.4 (6.5-6.6, resp.), of this proof in 6.1-6.6, we prove the nonexistence (existence, resp.) of nonconstant $\mathscr{A}$-harmonic measures with finite $p$-Dirichlet integrals for any elliptic operator 
$\mathscr{A} \in \mathscr{A}_{p}\left(B^{n}\right)$ with exponent $p$ in $[2, n]((1,2)$, resp.).

Thus in 6.1-6.4 we fix an arbitrary $p$ with $2 \leqq p \leqq n$ and an arbitrary elliptic operator $\mathscr{A} \in \mathscr{A}_{p}\left(B^{n}\right)$. In 6.1-6.3 we first give a direct proof (cf. [22] for an indirect one) for that there exist no nonconstant 2-Dirichlet finite 2-harmonic (i.e. the usual classical harmonic) measures on $B^{n}$ and then the assertion is generalized to an arbitrary $\mathscr{A}$-harmonic measures $\left(\mathscr{A} \in \mathscr{A}_{p}\left(B^{n}\right)\right)$ in 6.4.

Contrariwise suppose that there exists a nonconstant 2-Dirichlet finite 2-harmonic measure $w$ on $B^{n}$. By Lemma 3.2 the supremum (infimum, resp.) of $w$ on $B^{n}$ is 1 (0, resp.). By the Sard theorem (cf. e.g. [15; p. 16]) we can find two noncritical values $\lambda$ and $\mu$ of $w$ with $0<\lambda<\mu<1$ so that the level surfaces $\{w=\lambda\}$ and $\{w=\mu\}$ are smooth. We arbitrary choose and then fix a connected component $A$ of the open set $\{\lambda<w<\mu\}$ so that $A$ is a subregion of $B^{n}$ with smooth, possibly noncompact relative boundary $\partial_{B^{n}} A$ relative to $B^{n}$.

Observe that $w(1-w) \in W_{2,0}^{1}\left(B^{n}\right)$ by Lemma 3.1. Put $c=\lambda(1-\mu) / 4$ so that $0<c<1$. Since $W_{2,0}^{1}\left(B^{n}\right)$ is closed under lattice operations (cf. 1.3), we see that

$$
\chi=([w(1-w)] \cap c) / c
$$

again belongs to $W_{2,0}^{1}\left(B^{n}\right)$ along with $w(1-w)$ and $\chi=1$ in a neighborhood of $A$ $\cup \partial_{B^{n}} A$ in $B^{n}$. Since $\chi \in W_{2,0}^{1}\left(B^{n}\right)$, there exists a sequence $\left\{\chi_{k}\right\}$ in $C_{0}^{\infty}\left(B^{n}\right)$ such that

$$
\left\|\chi-\chi_{k} ; W_{2}^{1}\left(B^{n}\right)\right\| \rightarrow 0 \quad(k \rightarrow \infty)
$$

6.2. At this point it is convenient to consider $k$-forms $(k=0,1, \ldots, n)$

$$
\alpha=\alpha(x)=\sum_{i_{1}<\cdots<i_{k}} a_{i_{1} \cdots i_{k}}(x) d x^{i_{1}} \wedge \cdots \wedge d x^{i_{k}}
$$

on a ball $B=B(r)(0<r<\infty)$ as a Riemannian manifold with the Euclidean metric (cf. e.g. [5; p. 99]). We denote by $*$ the Hodge star operator with respect to the Euclidean metric on $B$ so that

$$
* \alpha=\sum_{j_{1}<\cdots<j_{n-k}} a_{j_{1} \cdots j_{n-k}}^{*}(x) d x^{j_{1}} \wedge \cdots \wedge d x^{j_{n-k}}
$$

where

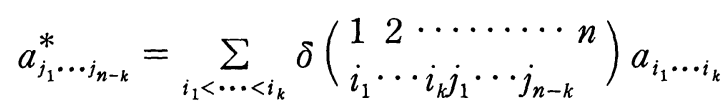

and 


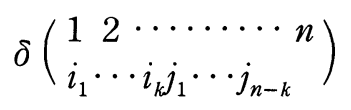

is the generalized Kronecker delta so that it is $1\left(-1\right.$, resp.) if $\left(i_{1}, \ldots, i_{k}, j_{1}, \ldots\right.$, $\left.j_{n-k}\right)$ is an even (odd, resp.) permutation of $(1, \ldots, n)$ and 0 if some two of $i_{1}, \ldots$, $i_{k}, j_{1}, \ldots, j_{n-k}$ are identical. We consider the pointwise inner product $\langle\alpha, \beta\rangle$ of $\alpha$ and $\beta$ on $B$ given by

$$
\langle\alpha, \beta\rangle=\langle\alpha(x), \beta(x)\rangle=\sum_{i_{1}<\cdots<i_{k}} a_{i_{1} \cdots i_{k}}(x) b_{i_{1} \cdots i_{k}}(x)
$$

where $\beta$ is also a $k$-form on $B$ with coefficients $b_{i_{1} \cdots i_{k}}$. Then the pointwise norm $|\alpha|$ of $\alpha$ on $B$ is given by

$$
|\alpha|^{2}=\langle\alpha, \alpha\rangle=\sum_{i_{1}<\cdots<i_{k}}\left(a_{i_{1} \cdots i_{k}}\right)^{2} .
$$

We will use the Schwarz ineguality: $|\langle\alpha, \beta\rangle| \leqq|\alpha||\beta|$. It is easily seen that

$$
\langle\alpha, \beta\rangle d x=\langle\alpha, \beta\rangle d x_{1} \wedge \cdots \wedge d x^{n}=\alpha \wedge * \beta
$$

where $d x=d x^{1} \cdots d x^{n}=d x^{1} \wedge \cdots \wedge d x^{n}$ is the volume element on the Euclidean ball $B$. We set

$$
(\alpha, \beta)=(\alpha, \beta)_{B}=\int_{B}\langle\alpha(x), \beta(x)\rangle d x=\int_{B} \alpha \wedge * \beta
$$

and

$$
\|\alpha\|^{2}=\|\alpha\|_{B}^{2}=(\alpha, \alpha)=\int_{B}|\alpha(x)|^{2} d x=\int_{B} \alpha \wedge * \alpha .
$$

If $\alpha$ is differentiable in the sense that $a_{i_{1} \cdots_{k}}$ are differentiable, then $d \alpha$ is defined as usual by

$$
d \alpha=\sum_{i_{1}<\cdots<i_{k}} d a_{i_{1} \cdots i_{k}} \wedge d x^{i_{1}} \wedge \cdots \wedge d x^{i_{k}}
$$

where $d f=\sum_{i=1}^{n}\left(\partial f / \partial x^{i}\right) d x^{i}$ for a differentiable function $f$ on $B$.

Now we return to the function $w$ introduced in 6.1 . Note that $d * d w=$ - $*(\nabla \cdot \nabla w)=-* \Delta w=0$ since $w$ is 2 -harmonic. This means that $* d w$ is a $C^{\infty}$ closed $(n-1)$-form on $B^{n}$. Then, by the Poincare lemma (cf. e.g. [11; p. $273])$, we can find a $C^{\infty}(n-2)$-form

$$
\alpha=\alpha(x)=\sum_{i_{1}<\cdots<i_{n-2}} a_{i_{1} \cdots i_{n-2}}(x) d x^{i_{1}} \wedge \cdots \wedge d x^{i_{n-2}}
$$


on $B^{n}$ such that $* d w=d \alpha$. Observe that

$$
\|d \alpha\|^{2}=\|* d w\|^{2}=\|d w\|^{2}=D_{2}\left(w ; B^{n}\right)<\infty
$$

based upon the fact that the Hodge $*$ operator is an isometry. Consider

$$
\alpha_{\rho}=\alpha_{\rho}(x)=\sum_{i_{1}<\cdots<i_{n-2}} a_{i_{1} \cdots i_{n-2}}(\rho x) d x^{i_{1}} \wedge \cdots \wedge d x^{i_{n-2}}
$$

for each $\rho$ in $(0,1)$, which is also a $C^{\infty}(n-2)$-form not only on $B^{n}$ but also on the larger ball $\{|x|<1 / \rho\}$ containing the closed ball $\bar{B}^{n}=\left\{x \in R^{n}:|x| \leqq 1\right\}$. Hence in particular the function

$$
\left|\alpha_{\rho}(x)\right|^{2}=\sum_{i_{1}<\cdots<i_{n-2}}\left(a_{i_{1} \cdots i_{n-2}}(\rho x)\right)^{2}
$$

is bounded on $B^{n}$. If we denote

$$
d \alpha(x)=\sum_{i_{1}<\cdots<i_{n-2}} d a_{i_{1} \cdots i_{n-2}}(x) \wedge d x^{i_{1}} \wedge \cdots \wedge d x^{i_{n-2}}
$$

by

$$
d \alpha(x)=\sum_{j_{1}<\cdots<j_{n-1}} b_{j_{1} \cdots j_{n-1}}(x) d x^{j_{1}} \wedge \cdots \wedge d x^{j_{n-1}},
$$

Then $b_{j_{1} \cdots j_{n-1}} \in L_{2}\left(B^{n}\right) \cap C^{\infty}\left(B^{n}\right)\left(j_{1}<\cdots<j_{n-1}\right)$ and

$$
d \alpha_{\rho}(x)=\sum_{\jmath_{1}<\cdots<j_{n-1}} \rho b_{j_{1} \cdots j_{n-1}}(\rho x) d x^{\jmath_{1}} \wedge \cdots \wedge d x^{\jmath_{n-1}} .
$$

Thus $d \alpha_{\rho}$ is $C^{\infty}$ on $\{|x|<1 / \rho\}$ that contains $\bar{B}^{n}$ and we have

$$
\begin{aligned}
& \left\|d \alpha-d \alpha_{\rho}\right\|^{2}=\int_{B^{n}}\left|d \alpha(x)-d \alpha_{\rho}(x)\right|^{2} d x \\
= & \sum_{j_{1}<\cdots<j_{n-1}} \int_{B^{n}}\left|b_{j_{1} \cdots j_{n-1}}(x)-\rho b_{j_{1} \cdots j_{n-1}}(\rho x)\right|^{2} d x \\
= & \sum_{j_{1}<\cdots<j_{n-1}}\left\|b_{j_{1} \cdots j_{n-1}}-\rho\left(b_{j_{1} \cdots j_{n-1}}\right)_{\rho} ; L_{2}\left(B^{n}\right)\right\|^{2}
\end{aligned}
$$

where $f_{\rho}(x)=f(\rho x)(0<\rho<1)$ for any function $f$ on $B^{n}$ as in 1.4. In order to conclude that

$$
\left\|d \alpha-d \alpha_{\rho}\right\| \rightarrow 0 \quad(\rho \uparrow 1)
$$

we only have to show that $\left\|f-\rho f_{\rho} ; L_{2}\left(B^{n}\right)\right\| \rightarrow 0 \quad(\rho \uparrow 1)$ for any function $f$ in $L_{2}\left(B^{n}\right)$. This follows from the simple inequality

$$
\left\|f-\rho f_{\rho} ; L_{2}\left(B^{n}\right)\right\| \leqq(1-\rho)\left\|f ; L_{2}\left(B^{n}\right)\right\|+\rho\left\|f-f_{\rho} ; L_{2}\left(B^{n}\right)\right\|
$$


and Corollary to Lemma 1.2: $\left\|f-f_{\rho} ; L_{2}\left(B^{n}\right)\right\| \rightarrow 0 \quad(\rho \uparrow 1)$ for any function $f$ in $L_{2}\left(B^{n}\right)$.

6.3. We denote by $(\cdot, \cdot)_{A}$ and $\|\cdot\|_{A}$ the inner product and the norm, respectively, considered for forms on the region $A$ introduced in 6.1 . If we simply write $(\cdot, \cdot)$ and $\|\cdot\|$, then they are considered on $B^{n}$.

To compute $\left(d w, * d\left(\chi_{k} \alpha_{\rho}\right)\right)_{A}$ for any $\rho$ in $(0,1)$ and any integer $k \geqq 1$, we choose a ball $B=B(r)(0<r<1)$ containing the support of $\chi_{k}$, where $\left\{\chi_{k}\right\}$ is the sequence in $C_{0}^{\infty}\left(B^{n}\right)$ introduced in 6.1 . Then, by the Stokes formula, we compute as follows:

$$
\begin{gathered}
\left(d w, * d\left(\chi_{k} \alpha_{\rho}\right)\right)_{A}=(-1)^{n-1} \int_{A} d w \wedge d\left(\chi_{k} \alpha_{\rho}\right)=\int_{A \cap B} d\left(\chi_{k} \alpha_{\rho}\right) \wedge d w \\
=\int_{\partial(A \cap B)} \chi_{k} \alpha_{\rho} \wedge d w=\left(\int_{(\partial A) \cap B}+\int_{A \cap \partial B}\right) \chi_{k} \alpha_{\rho} \wedge d w
\end{gathered}
$$

where the boundary operator $\partial=\partial_{B^{n}}$ is considered with respect to $B^{n}$. The first term on the right most side of the above vanishes because $d w=0$ along $\partial A$, and the second also vanishes because $\chi_{k}=0$ on $\partial B$. Thus we can conclude that

$$
\left(d w, * d\left(\chi_{k} \alpha_{\rho}\right)\right)_{A}=0
$$

for every $\rho$ in $(0,1)$ and every integer $k \geqq 1$.

Next we compute $\left(d w, * d \alpha_{\rho}\right)_{A}$ for any fixed $\rho$ in $(0,1)$. For any integer $k \geqq 1$ we evaluate $\left|\left(d w, * d \alpha_{\rho}\right)_{A}\right|$ as follows:

$$
\begin{aligned}
& \left|\left(d w, * d \alpha_{\rho}\right)_{A}\right|=\left|\left(d w, * d \alpha_{\rho}\right)_{A}-\left(d w, * d\left(\chi_{k} \alpha_{\rho}\right)\right)_{A}\right| \\
& =\left|\left(d w, * d\left(\chi \alpha_{\rho}\right)\right)_{A}-\left(d w, * d\left(\chi_{k} \alpha_{\rho}\right)\right)_{A}\right| \\
& \leqq\|d w\|_{A}\left\|d\left(\left(\chi-\chi_{k}\right) \alpha_{\rho}\right)\right\|_{A} \leqq\|d w\| \| d\left(\left(\chi-\chi_{k}\right) \alpha_{\rho} \| .\right.
\end{aligned}
$$

Here the fact that the Hodge $*$ operator is an isometry is again used. We further estimate the second factor of the term on the right most side of the above inequalities. Since

$$
d\left(\left(\chi-\chi_{k}\right) \alpha_{\rho}\right)=\left(d \chi-d \chi_{k}\right) \wedge \alpha_{\rho}+\left(\chi-\chi_{k}\right) d \alpha_{\rho}
$$

we see that

$$
\begin{gathered}
\left|\left(d w, * d \alpha_{\rho}\right)_{A}\right| \\
\leqq\|d w\|\left(\left(\int_{B^{n}}\left|\left(d \chi-d \chi_{k}\right) \wedge \alpha_{\rho}\right|^{2} d x\right)^{1 / 2}+\left(\int_{B^{n}}\left|\left(\chi-\chi_{k}\right) d \alpha_{\rho}\right|^{2} d x\right)^{1 / 2}\right) .
\end{gathered}
$$


Since $\alpha_{\rho}$ and $d \alpha_{\rho}$ are $C^{\infty}$ on $\{|x|<1 / \rho\}$ containing $\bar{B}^{n}$, there exists a positive constant $K_{\rho}$ such that

$$
\left|\left(d \chi-d \chi_{k}\right) \wedge \alpha_{\rho}\right|^{2} \leqq K_{\rho}^{2}\left|d \chi-d \chi_{k}\right|^{2}=K_{\rho}^{2}\left|\nabla \chi-\nabla \chi_{k}\right|^{2}
$$

and

$$
\left|\left(\chi-\chi_{k}\right) d \alpha_{\rho}\right|^{2} \leqq K_{\rho}^{2}\left|\chi-\chi_{k}\right|^{2}
$$

on $B^{n}$. Therefore we obtain that

$$
\begin{gathered}
\left|\left(d w, * d \alpha_{\rho}\right)_{A}\right| \leqq\|d w\|\left(K_{\rho}\left(\int_{B^{n}}\left|\nabla \chi-\nabla \chi_{k}\right|^{2} d x\right)^{1 / 2}+K_{\rho}\left(\int_{B^{n}}\left|\chi-\chi_{k}\right|^{2} d x\right)^{1 / 2}\right) \\
=K_{\rho}\|d w\|\left(\left\|\nabla \chi-\nabla \chi_{k} ; L_{2}\left(B^{n}\right)^{n}\right\|+\left\|\chi-\chi_{k} ; L_{2}\left(B^{n}\right)\right\|\right) \\
=K_{\rho}\|d w\|\left\|\chi-\chi_{k} ; W_{2}^{1}\left(B^{n}\right)\right\| \rightarrow 0 \quad(k \uparrow \infty)
\end{gathered}
$$

by (6.1). We have thus deduced that

$$
\left(d w, * d \alpha_{\rho}\right)_{A}=0
$$

for every $\rho$ in $(0,1)$.

Finally we see by recalling $* d w=d \alpha$ and the isometry of the Hodge * operator that

$$
\begin{gathered}
\|d w\|_{A}^{2}=(d w, d w)_{A}=\left(d w,(-1)^{n-1} * d \alpha\right)_{A}=\left|(d w, * d \alpha)_{A}\right| \\
=\left|(d w, * d \alpha)_{A}-\left(d w, * d \alpha_{\rho}\right)_{A}\right| \leqq\|d w\|_{A}\left\|d \alpha-d \alpha_{\rho}\right\|_{A} \\
\leqq\|d w\|\left\|d \alpha-d \alpha_{\rho}\right\| \rightarrow 0 \quad(\rho \uparrow 1)
\end{gathered}
$$

by (6.2). Hence $\|d w\|_{A}=0$ and $w$ is a constant on $A$. By the unicity theorem for 2 -harmonic functions, we see that $w$ is a constant on $B^{n}$, which contradicts our original assumption made in 6.1 that $w$ is nonconstant on $B^{n}$.

We have thus established that there exist no nonconstant 2-Dirichlet finite 2-harmonic measures on $B^{n}$.

6.4. Given an arbitrary elliptic operator $\mathscr{A}: B^{n} \times R^{n} \rightarrow R^{n}$ of exponent $p$ in $2 \leqq p \leqq n$, i.e. $\mathscr{A} \in \mathscr{A}_{p}\left(B^{n}\right)$ (cf. 2.1). We now maintain that there exist no nonconstant $p$-Dirichlet finite $\mathscr{A}$-harmonic measures on $B^{n}$.

For this aim we take any $p$-Dirichlet finite $\mathscr{A}$-harmonic measure $w$ on $B^{n}$ so that $w \in W_{p}^{1}\left(B^{n}\right)$. We are to show that $w$ is a constant. Since $2 \leqq p$, we have

$$
W_{2}^{1}\left(B^{n}\right) \supset W_{p}^{1}\left(B^{n}\right)
$$

and similarly 


$$
W_{2,0}^{1}\left(B^{n}\right) \supset W_{p, 0}^{1}\left(B^{n}\right)
$$

as we saw in 1.7. By Lemma 3.1 we have that

$$
w(1-w) \in W_{p, 0}^{1}\left(B^{n}\right) .
$$

Hence by the above inclusion relation we also have that

$$
w(1-w) \in W_{2,0}^{1}\left(B^{n}\right) .
$$

By Lemma 3.3, the 2-harmonic part $\pi_{2}^{B^{n}} w$ of $w \in W_{2}^{1}\left(B^{n}\right)$ is a 2-Dirichlet finite 2 -harmonic measure on $B^{n}$, which must be a constant $c$ on $B^{n}$ as we have shown in 6.1-6.3. Thus we have the decomposition

$$
w=c+g \quad\left(g \in W_{2,0}^{1}\left(B^{n}\right)\right) .
$$

Since actually $w \in W_{p}^{1}\left(B^{n}\right)$ and trivially $c \in W_{p}^{1}\left(B^{n}\right)$, we see that $g \in$ $W_{p}^{1}\left(B^{n}\right)$ and hence $g \in W_{2,0}^{1}\left(B^{n}\right) \cap W_{p}^{1}\left(B^{n}\right)$. Recall that

$$
W_{2,0}^{1}\left(B^{n}\right) \cap W_{p}^{1}\left(B^{n}\right)=W_{p, 0}^{1}\left(B^{n}\right)
$$

(cf. (1.6)). Therefore we have the decomposition (2.11) for $w$ on $B^{n}$ :

$$
w=c+g \quad\left(c \in W_{p, \mathscr{A}}^{1}\left(B^{n}\right), g \in W_{p, 0}^{1}\left(B^{n}\right)\right) .
$$

On the other hand

$$
w=w+0 \quad\left(w \in W_{p, \mathscr{A}}^{1}\left(B^{n}\right), 0 \in W_{p, 0}^{1}\left(B^{n}\right)\right)
$$

is another decomposition of $w$ in (2.11) and the uniqueness of the decomposition implies that $w=\pi_{\mathscr{A}}^{B^{n}} w=c$, a constant. We have thus shown that $w$ is a constant.

6.5. Given an arbitrary $p$ in $1<p<2$ and an arbitrary elliptic operator $\mathscr{A}: B^{n} \times R^{n} \rightarrow R^{n}$ of exponent $p$, i.e. $\mathscr{A} \in \mathscr{A}_{p}\left(B^{n}\right)$. We will show that there exists a nonconstant $p$-Dirichlet finite $\mathscr{A}$-harmonic measure on $B^{n}$.

For the purpose we consider two surfaces $\gamma_{0}: x^{n}=0$ and $\gamma_{1}: x^{n}=2^{-1}(1-$ ,$\left.x^{\prime} \mid\right)$ in $B^{n}$ where we denote $x=\left(x^{1}, \ldots, x^{n-1}, x^{n}\right)$ by $\left(x^{\prime}, x^{n}\right)$ so that $x^{\prime}=\left(x^{1}\right.$, $\left.\ldots, x^{n-1}\right)$. Note that $\gamma_{0}$ may be identified with the ball $B^{n-1}$ in the subspace $\{x \in$ $\left.R^{n}: x^{n}=0\right\}$ which is viewed as the $(n-1)$-dimensional Euclidean space $R^{n-1}$. The ball $B^{n}$ is divided into three parts $B_{i}(i=0,1,2): B_{0}\left(B_{1}\right.$, resp. $)$ is the part of $B^{n}$ below $\gamma_{0}$ (above $\gamma_{1}$, resp.); $B_{2}$ is the part between $\gamma_{0}$ and $\gamma_{1}$. We consider a function

$$
g(x)= \begin{cases}j & \left(x \in B_{j}, j=0,1\right), \\ 2 x^{n} /\left(1-\left|x^{\prime}\right|\right) & \left(x \in B_{2}\right)\end{cases}
$$


on $B^{n}$. Clearly $g \in C\left(B^{n}\right) \cap L_{\infty}\left(B^{n}\right)$ and $g$ is absolutely continuous on all lines in $B^{n}$ parallel to coordinate axes. We will see below that the ordinary gradient $\nabla g$ of $g$ is $p^{\text {th }}$ integrable on $B^{n}$. Hence by the Nikodym theorem (cf. 1.3) we can conclude that $g \in W_{p}^{1}\left(B^{n}\right)$.

The ordinary gradient $\nabla g$ of $g$ is given as follows:

$$
\nabla g(x)= \begin{cases}0 & \left(x \in B_{0} \cup B_{1}\right), \\ \left(\nabla_{x^{\prime}} g(x), 2 /\left(1-\left|x^{\prime}\right|\right)\right. & \left(x \in B_{2} \backslash\left\{x^{\prime}=0\right\}\right)\end{cases}
$$

where $\nabla_{x^{\prime}}=\left(\partial / \partial x^{1}, \ldots, \partial / \partial x^{n-1}\right)$ so that

$$
\nabla_{x^{\prime}} g(x)=2 x^{n}\left(1-\left|x^{\prime}\right|\right)^{-2}\left(x^{1} /\left|x^{\prime}\right|, \ldots, x^{n-1} /\left|x^{\prime}\right|\right)
$$

for $x \in B_{2} \backslash\left\{x^{\prime}=0\right\}$. Observe that

$$
\left\|\nabla g ; L_{p}\left(B^{n}\right)^{n}\right\|=\left\|\nabla g ; L_{p}\left(B_{2}\right)^{n}\right\| .
$$

Here by

$$
\begin{gathered}
|\nabla g|^{p}=\left(\left|\nabla_{x^{\prime}} g\right|^{2}+\left|\partial g / \partial x^{n}\right|^{2}\right)^{p / 2} \\
\leqq\left(\left|\nabla_{x^{\prime}} g\right|+\left|\partial g / \partial x^{n}\right|\right)^{p} \leqq 2^{p}\left(\left|\nabla_{x^{\prime}} g\right|^{p}+\left|\partial g / \partial x^{n}\right|^{p}\right)
\end{gathered}
$$

we see that

$$
\left\|\nabla g ; L_{p}\left(B_{2}\right)^{n}\right\| \leqq 2^{p} \int_{B_{2}}\left(\left|\nabla_{x^{\prime}} g(x)\right|^{p}+\left|\partial g(x) / \partial x^{n}\right|^{p}\right) d x .
$$

The right hand side of the above equals, by the Fubini theorem,

$$
\begin{gathered}
4^{p} \int_{B^{n-1}}\left(\int_{0}^{\left(1-\left|x^{\prime}\right|\right) / 2}\left(\left(x^{n}\right)^{p} /\left(1-\left|x^{\prime}\right|\right)^{2 p}+1 /\left(1-\left|x^{\prime}\right|\right)^{p}\right) d x^{n}\right) d x^{\prime} \\
=c \int_{B^{n-1}}\left(1-\left|x^{\prime}\right|\right)^{1-p} d x^{\prime}
\end{gathered}
$$

where $c=4^{p}\left(1 / 2^{p+1}(p+1)+1 / 2\right)$. Observe that $\sum^{n}=\partial B^{n} \cap\left\{x^{n}=0\right\}$ (cf. $4.1)$ is the boundary $\partial B^{n-1}$ of $B^{n-1}$ considered in the $(n-1)$-dimensional subspace $\left\{x^{n}=0\right\}$ of $R^{n}$. If we denote by $d \omega_{n-1}$ the area element on $\partial B^{n-1}$, then we have

$$
\begin{gathered}
\int_{B^{n-1}}\left(1-\left|x^{\prime}\right|\right)^{1-p} d x^{\prime}=\int_{\partial B^{n-1}}\left(\int_{0}^{1}(1-r)^{1-p} r^{n-2} d r\right) d \omega_{n-1} \\
\leqq\left|\partial B^{n-1}\right| \int_{0}^{1}(1-r)^{1-p} d r=\left|\partial B^{n-1}\right| /(2-p)<\infty
\end{gathered}
$$


where $\left|\partial B^{n-1}\right|$ is the area of $\partial B^{n-1}$ under the convention of $\left|\partial B^{1}\right|=2$ with $d \omega_{1}$ $=d \delta_{e}+d \delta_{-e}(e=(1,0))$ when $n=2$. Here the assumption $1<p<2$ is essentially made use of. Thus $\left\|\nabla g ; L_{p}\left(B^{n}\right)^{n}\right\|<\infty$ and we have established that $g \in$ $W_{p}^{1}\left(B^{n}\right)$. Since $g \in C\left(B^{n}\right) \cap L_{\infty}\left(B^{n}\right)$, we actually have that $g \in M_{p}\left(B^{n}\right)$.

One step further we maintain that

$$
g(1-g) \in W_{p, 0}^{1}\left(B^{n}\right)
$$

in addition to $g(1-g) \in W_{p}^{1}\left(B^{n}\right)$ already established above. For simplicity we set $h=g(1-g) \in W_{p}^{1}\left(B^{n}\right)$. Take the sequence $\left\{\chi_{k}\right\}$ in Lemma 4.1 and set

$$
h_{k}=\chi_{k} h \quad(k=1,2, \ldots) \text {. }
$$

(Do not be confused with the sequence $\left\{\chi_{k}\right\}$ constructed in 6.1 and used in 6.3.) Clearly $h_{k}$ belongs to $W_{p}^{1}\left(B^{n}\right)$ and the support of $h_{k}$ is contained in $B^{n}$ so that we see that $h_{k} \in W_{p, 0}^{1}\left(B^{n}\right)$. Therefore we can conclude that $h \in W_{p, 0}^{1}\left(B^{n}\right)$ if

$$
\left\|h-h_{k} ; W_{p}^{1}\left(B^{n}\right)\right\|=\left\|\varphi_{k} h ; W_{p}^{1}\left(B^{n}\right)\right\| \rightarrow 0 \quad(k \uparrow \infty)
$$

is assured, where $\varphi_{k}=1-\chi_{k}$. To see this we first note that

$$
\left\|\varphi_{k} h ; L_{p}\left(B^{n}\right)\right\|^{p}=\int_{B^{n}}\left|\varphi_{k}(x) h(x)\right|^{p} d x \rightarrow 0 \quad(k \uparrow \infty)
$$

by the Lebesgue dominated convergence theorem since the integrand is dominated by the integrable function $|h(x)|^{p}$ and $\varphi_{k} \rightarrow 0 \quad(k \uparrow \infty)$ on $B^{n}$ (cf. Lemma 4.1). Next observe that

$$
\begin{gathered}
\left\|\nabla\left(\varphi_{k} h\right) ; L_{p}\left(B^{n}\right)^{n}\right\| \\
\leqq\left(\int_{B^{n}}|h(x)|^{p}\left|\nabla \varphi_{k}(x)\right|^{p} d x\right)^{1 / p}+\left(\int_{B^{n}}\left|\varphi_{k}(x)\right|^{p}|\nabla h(x)|^{p} d x\right)^{1 / p} .
\end{gathered}
$$

The first term of the right hand side of the above inequality is dominated by

$$
\left\|h ; L_{\infty}\left(B^{n}\right)\right\| D_{p}\left(\varphi_{k} ; B^{n}\right)^{1 / p} \leqq 2^{-k}\left\|h ; L_{\infty}\left(B^{n}\right)\right\| \rightarrow 0 \quad(k \uparrow \infty)
$$

(cf. Lemma 4.1) and the second term by

$$
\left(\int_{T^{n}\left(t_{k}\right)}|\nabla h(x)|^{p} d x\right)^{1 / p} \rightarrow\left(\int_{\Sigma^{n}}|\nabla h(x)|^{p} d x\right)^{1 / p}=0 \quad(k \uparrow \infty)
$$

(cf. Lemma 4.1) since $\left|\sum^{n}\right|=0$. We have thus completed the proof of (6.5) and hence of (6.4). 
6.6. Finally take the $\mathscr{A}$-harmonic part $w=\pi_{\mathscr{A}}^{B^{n}} g$ of $g$ on $B^{n}$. By Lemma 3.3 and (6.4) we see that $w$ is a $p$-Dirichlet finite $\mathscr{A}$-harmonic measure on $B^{n}$. The proof will be over if we show that $w$ is nonconstant on $B^{n}$. Contrariwise we assume that $w$ is a constant $c$ on $B^{n}$. Then $g-c \in W_{p, 0}^{1}\left(B^{n}\right)$. Observe that the particular function

$$
v(x)=v\left(x^{1}, \ldots, x^{n}\right)=x^{n}
$$

is a $p$-Dirichlet finite $p$-harmonic function on $B^{n}$ in the genuine sense:

$$
\nabla \cdot\left(|\nabla v|^{p-2} \nabla v\right)=\nabla \cdot(0, \ldots, 0,1)=0 .
$$

Hence in particular $v$ is a weak solution of $\nabla \cdot\left(|\nabla u|^{p-2} \nabla u\right)=0$ so that

$$
\int_{B^{n}}|\nabla v(x)|^{p-2} \nabla v(x) \cdot \nabla g(x) d x=\int_{B^{n}}|\nabla v(x)|^{p-2} \nabla v(x) \cdot \nabla(g(x)-c) d x=0
$$

since $g-c \in W_{p, 0}^{1}\left(B^{n}\right)$. On the other hand, by $\nabla v=(0, \ldots, 0,1)$ and $(6.3)$, we must have, based on the Fubini theorem, that

$$
\begin{aligned}
& \int_{B^{n}}|\nabla v(x)|^{p-2} \nabla v(x) \cdot \nabla g(x)=\int_{B_{2}} 2\left(1-\left|x^{\prime}\right|\right)^{-1} d x \\
= & \int_{B^{n-1}}\left(\int_{0}^{\left(1-\left|x^{\prime}\right|\right) / 2} 2\left(1-\left|x^{\prime}\right|\right)^{-1} d x^{n}\right) d x^{\prime}=\left|B^{n-1}\right|_{R^{n-1}}>0,
\end{aligned}
$$

where $\left|B^{n-1}\right|_{R^{n-1}}$ is the volume of $B^{n-1}$ considered in the space $R^{n-1}$, and thus we arrived at a contradiction. Therefore $w$ is nonconstant.

The proof of the main theorem in the analytic form is herewith complete.

\section{REFERENCES}

[1] R. Accola, The bilinear relation on open Riemann surfaces, Trans. Amer. Math. Soc., 96 (1960), 143-161.

[2] R. A. Adams, Sobolev Spaces, Academic Press, 1978.

[3] L. Ahlfors and L. Sario, Riemann Surfaces, Princeton, 1960.

[4] C. Constantinescu und A. Cornea, Ideale Ränder Riemannscher Flächen, Springer, 1963.

[5] G. de Rham, Variété, Différentiables, Hermann, 1955.

[6] N. Dunford and J. T. Schwartz, Linear Operators, Part I, Interscience, 1967.

[7] M. Glasner and M. Nakai, Riemannian manifolds with discontinuous metrics and the Dirichlet integrals, Nagoya Math. J., 46 (1972), 1-48.

[8] M. Heins, On the Lindelöf principle, Ann. Math., 61 (1955), 440-473.

[9] I. Holopainen, Nonlinear potential theory and quasiregular mappings on Riemannian manifolds, Ann. Acad. Sci. Fenn., Ser. AI Math. Dissertation, 74 (1990), 1-45.

[10] H. Ishida, Harmonic Dirichlet functions and the components of harmonic bound- 
aries of Riemann surfaces, J. Math. Kyoto Univ., 29 (1989), 625-641.

[11] S. Krantz, Function Theory of Several Complex Variables, Wiley, 1982.

[12] Y. Kusunoki, Characterizations of canonical differentials, J. Math. Kyoto Univ., 5 (1966), 197-207.

[13] J. Lelong-Ferrand, Etude d'une class d'application liées à des homomorphismes d'algebres de fonctions, et generalisant les quasi conformes, Duke Math. J., 40 (1973), 163-186.

[14] L. G. Lewis, Quasiconformal mappings and Royden Algebras in space, Trans. Amer. Math. Soc., 158 (1971), 481-492.

[15] J. Milnor, Topology from the Differentiable Viewpoint, Univ. Press of Va., 1965.

[16] S. Mizohata, The Theory of Partial Differential Equations, Cambridge Univ. Press, 1973.

[17] V. G. Maz'ja, On the continuity at a boundary point of solutions of quasi-linear elliptic equations, Vestnik Leningrad Univ., 3 (1976), 225-242.

[18] V. G. Maz'ja, Sobolev Spaces, Springer, 1985.

[19] M. Nakai, "On parabolicity and Royden compactifications of Riemannian manifolds" in Proceedings of the International Conference on Functional Analysis and Related Topics, 1969, 316-325.

[20] M. Nakai, Royden algebras and quasi-isometries of Riemannian manifolds, Pacific J. Math., 40 (1972), 397-414.

[21] M. Nakai, Dirichlet finite harmonic measures on Riemann surfaces, Complex Variables, 17 (1991), 123-131.

[22] M. Nakai, Riemannian manifolds with connected Royden harmonic boundaries, Duke Math. J., 67 (1992), 589-625.

[23] M. Nakai, Existence of Dirichlet finite harmonic measures in nonlinear potential theory, Complex Variables, 21 (1993), 107-114.

[24] M. Nakai and H. Tanaka, Existence of quasiconformal mappings between Riemannian manifolds, Kodai Math. J., 5 (1982), 122-131.

[25] M. Ohtsuka, Extremal Length and p-precise Functions (In preparation).

[26] Yu. G. Reshetnyak, Space Mappings with Bounded Distortion, Amer. Math. Soc., 1989.

[27] W. Rudin, Real and Complex Analysis, McGraw-Hill, 1987.

[28] L. Sario and M. Nakai, Classification Theory of Riemann Surfaces, Springer, 1970.

[29] J. Serrin, Local behavior of solutions of quasilinear equations, Acta Math., 111 (1964), 247-302.

[30] J. Serrin, Isolated singularities of solutions of quasilinear equations, Acta Math., 113 (1965), 219-240.

[31] N. Sonderborg, Quasiregular mappings with finite multiplicity and Royden algebras, Indiana Univ. Math. J., 40 (1991), 1143-1167.

[32] H. Tanaka, Harmonic boundaries of Riemannian manifolds, Nonlinear Anal., 14 (1990), 55-67.

Department of Mathematics

Nagoya Institute of Technology

Gokiso, Showa, Nagoya 466

Japan 